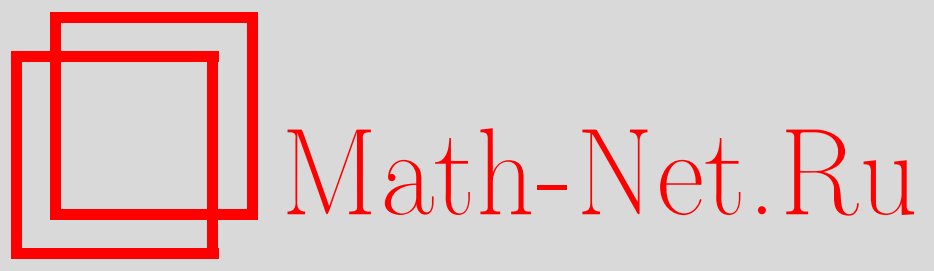

А. В. Устинов, Минимальные системы векторов в трехмерных решетках и аналог теоремы Валена для трехмерных цепных дробей Минковского, Совр. пробл. матем., 2012, выпуск 16, 103-128

DOI: https://doi.org/10.4213/spm38

Использование Общероссийского математического портала Math-Net.Ru подразумевает, что вы прочитали и согласны с пользовательским соглашением http: //www . mathnet.ru/rus/agreement

Параметры загрузки:

IP : 3.95 .254 .165

26 апреля 2023 г., $14: 17: 47$ 


\title{
Минимальные системы векторов в трехмерных решетках и аналог теоремы Валена для трехмерных цепных дробей Минковского
}

\author{
А. В. Устинов \\ Хабаровское отделение Института прикладной математики ДВО РАН
}

Памяти А. А. Карацубы

\section{1. Введение}

Существуют две геометрические интерпретации классических цепных дробей, которые допускают естественное обобщение на многомерный случай. В первой из них, принадлежащей Клейну (см. [1], [2]), цепная дробь идентифицируется с выпуклой оболочкой (многоугольником Клейна) точек целочисленной решетки, лежащих в двух смежных углах. Вторая интерпретация, независимо предложенная Вороным и Минковским (см. [3]-[6]), основана на использовании локальных минимумов решеток, минимальных систем и экстремальных параллелепипедов (определения использованных во введении понятий будут даны ниже). Вершины многоугольников Клейна в плоских решетках можно отождествить с локальными минимумами, однако начиная с размерности 3 геометрические конструкции Клейна и ВороногоМинковского становятся различными (см. [7], [8]).

Конструкции Вороного и Минковского более просты с вычислительной точки зрения. В частности, они позволяют строить эффективные алгоритмы нахождения основных единиц в кубических полях. Трехмерная теория цепных дробей как у Вороного, так и у Минковского базируется на красивых теоремах из геометрии чисел (см. обсуждение и переизложение оригинальных результатов в монографиях [9]-[11]). Конструкции Вороного и Минковского строятся в рамках естественного предположения, что мы имеем дело с неприводимыми решетками (у разных точек решетки не может совпадать ни одна из координат). В частности, этим свойством обладают решетки кубических иррациональностей. Однако в ряде теоретико-числовых задач, например, при анализе свойств сеток Коробова (см. [12]-[14]) возникает необходимость в изучении локальных свойств целочисленных решеток, которые не являются неприводимыми.

В статье [15] отмечено, что теорема Валена о приближении чисел подходящими дробями (см. [16], [17]) в терминах решеток имеет следующую интерпретацию: если векторы $\gamma_{a}=$ $\left(a_{1}, a_{2}\right), \gamma_{b}=\left(-b_{1}, b_{2}\right)$ образуют базис Вороного, то

$$
\min \left\{a_{1} a_{2}, b_{1} b_{2}\right\} \leqslant \frac{1}{2} \operatorname{det} \Gamma
$$

В той же статье было предложено уточнение теоремы Валена:

$$
a_{1} a_{2}+b_{1} b_{2} \leqslant \operatorname{det} \Gamma
$$

Работа выполнена при поддержке Российского фонда фундаментальных исследований (грант № 11-0100628-а), программы "Ведущие научные школы" (грант № НШ-1922.2012.1), проекта ДВО № 12-I-П19-01 и фонда «Династия». 
В работе [15] Авдеевой и Быковским был доказан аналог неравенства (1.1) для базисов Минковского (трехмерных аналогов базисов Вороного): если узлы

$$
\gamma_{a}=\left( \pm a_{1}, \pm a_{2}, \pm a_{3}\right), \quad \gamma_{b}=\left( \pm b_{1}, \pm b_{2}, \pm b_{3}\right), \quad \gamma_{c}=\left( \pm c_{1}, \pm c_{2}, \pm c_{3}\right)
$$

где $a_{i}, b_{i}, c_{i} \geqslant 0, i=1,2,3$, составляют базис Минковского решетки $\Gamma$, то

$$
a_{1} a_{2} a_{3}+b_{1} b_{2} b_{3}+c_{1} c_{2} c_{3} \leqslant \operatorname{det} \Gamma
$$

(Предыдущие результаты, связанные с трехмерной теоремой Валена, а также некоторые ее уточнения могут быть найдены в [15], [18]-[21].) Неравенство (1.3) можно рассматривать как уточнение оценки

$$
a_{1} a_{2} a_{3}+b_{1} b_{2} b_{3}+c_{1} c_{2} c_{3} \leqslant 3 \operatorname{det} \Gamma,
$$

следующей из теоремы Минковского о выпуклом теле.

В настоящей статье предлагается подход к изучению минимальных систем векторов в произвольных решетках. В приводимых решетках понятию минимальности можно придавать разный смысл, накладывая более или менее сильные условия на системы векторов. Разные ограничения приводят к разным вариантам трехмерного аналога теоремы Валена. В рамках предлагаемого подхода для минимальных систем вида (1.2) будет выполняться ослабленный вариант теоремы Валена

$$
a_{1} a_{2} a_{3}+b_{1} b_{2} b_{3}+c_{1} c_{2} c_{3} \leqslant 2 \operatorname{det} \Gamma,
$$

в то время как для вполне минимальных систем (удовлетворяющих более сильным ограничениям) останется справедливой более точная оценка (1.3). Этот результат будет основан на полной классификации минимальных систем векторов в произвольных трехмерных решетках.

\section{2. Минимальные системы и базисы Минковского}

2.1. Основные понятия. Пусть $\gamma_{1}, \ldots, \gamma_{t}$ - произвольный набор линейно независимых векторов в пространстве $\mathbb{R}^{s}, 1 \leqslant t \leqslant s$. Решеткой размерности $s$ и ранга $t$ называется множество

$$
\Gamma=\left\langle\gamma_{1}, \ldots, \gamma_{t}\right\rangle:=\left\{m_{1} \gamma_{1}+\cdots+m_{t} \gamma_{t}: m_{1}, \ldots, m_{t} \in \mathbb{Z}\right\}
$$

Если $t=s$, то решетка называется полной. Для полной решетки Г величина

$$
\operatorname{det} \Gamma=\left|\operatorname{det}\left(\gamma_{1}, \ldots, \gamma_{s}\right)\right|
$$

не зависит от выбора базиса $\left(\gamma_{1}, \ldots, \gamma_{s}\right)$ и называется определителем решетки. Если $v_{1}, \ldots, v_{s}-$ точки (узлы) решетки $Г$, то целое число

$$
I=I\left(v_{1}, \ldots, v_{s}\right)=\frac{\left|\operatorname{det}\left(v_{1}, \ldots, v_{s}\right)\right|}{\left|\operatorname{det}\left(\gamma_{1}, \ldots, \gamma_{s}\right)\right|}=\frac{\left|\operatorname{det}\left(v_{1}, \ldots, v_{s}\right)\right|}{\operatorname{det} \Gamma}
$$

называется индексом набора $\left(v_{1}, \ldots, v_{s}\right)$ в решетке Г. В частности, равенство $I=1$ равносильно тому, что $\left(v_{1}, \ldots, v_{s}\right)$ - базис решетки $\Gamma ; I=0$ тогда и только тогда, когда $\left(v_{1}, \ldots, v_{s}\right)-$ вырожденный (линейно зависимый) набор векторов.

Решетка Г называется неприводимой (или решеткой общего положения), если координатные гиперплоскости не содержат узлов этой решетки, отличных от начала координат; в противном случае решетка называется приводимой. Множество полных $s$-мерных решеток будем обозначать через $\mathcal{L}_{s}(\mathbb{R})$, а его подмножество, состоящее из неприводимых решеток, через $\mathcal{L}_{s}^{*}(\mathbb{R})$. 
Для непустого точечного множества $T \subset \mathbb{R}^{s}$ положим

$$
\begin{aligned}
|T|_{i}= & \max \left\{\left|x_{i}\right|: x=\left(x_{1}, \ldots, x_{s}\right) \in T\right\}, \quad i=1, \ldots, s, \\
& \Pi(T)=\left(-|T|_{1},|T|_{1}\right) \times \cdots \times\left(-|T|_{s},|T|_{s}\right), \\
& \bar{\Pi}(T)=\left[-|T|_{1},|T|_{1}\right] \times \cdots \times\left[-|T|_{s},|T|_{s}\right] .
\end{aligned}
$$

Системой узлов порядка $r$ решетки Г (не обязательно полной) назовем любой конечный набор ненулевых узлов решетки $\left(\gamma_{1}, \ldots, \gamma_{r}\right)$, в котором $\gamma_{i} \neq \pm \gamma_{j}, 1 \leqslant i<j \leqslant r$. Произвольной системе $S=\left(\gamma_{1}, \ldots, \gamma_{r}\right)$ будем ставить в соответствие матрицу $M\left(\gamma_{1}, \ldots, \gamma_{r}\right)$, записывая координаты векторов $\gamma_{1}, \ldots, \gamma_{r}$ по столбцам.

Пусть $G_{s}$ - группа, действующая на множестве матриц вида $M\left(\gamma_{1}, \ldots, \gamma_{r}\right)$, которая порождена следующими элементарными преобразованиями:

1) перестановкой строк или столбцов (переобозначением векторов данной системы или осей координат);

2) изменением знаков в столбце (изменением направления оси координат);

3) умножением строки на ненулевое число (изменением масштаба на одной из координатных осей с возможным изменением ее ориентации).

Отметим, что при домножении строк матрицы системы векторов некоторой решетки на число, отличное от \pm 1 , то же преобразование нужно сделать и с матрицей базиса решетки.

2.2. Минимальные системы в неприводимых решетках. Локальные свойства приводимых и неприводимых решеток могут существенно отличаться. Остановимся сначала на решетках общего положения, поскольку их свойства значительно проще.

Узел $\gamma$ решетки $\Gamma \in \mathcal{L}_{s}(\mathbb{R})$ называется относительным (локальным) минимумом решетки Г по Вороному (в дальнейшем - просто минимумом), если замкнутый параллелепипед $\bar{\Pi}(\gamma)$ не содержит узлов решетки $\Gamma$, отличных от своих вершин и начала координат (см. [4]).

Пусть $Г$ - неприводимая, не обязательно полная решетка произвольной размерности. Система $S$ решетки Г называется минимальной, если параллелепипед $\Pi(S)$ не содержит узлов решетки $\Gamma$, отличных от начала координат. В частности, для неприводимых решеток понятия «минимальная система порядка 1» и «локальный минимумум» совпадают. Для приводимых решеток определение минимальной системы будет нуждаться в уточнении.

Для минимальной системы $S$ параллелепипед $\Pi(S)$ называется экстремальным: ни по одной из координат его нельзя расширить так, чтобы он по-прежнему не содержал ненулевых узлов решетки.

Если $\gamma \in \Pi\left(\gamma_{1}, \ldots, \gamma_{t}\right)$, то будем говорить, что узел $\gamma$ нарушает минимальность системь $\left(\gamma_{1}, \ldots, \gamma_{t}\right)$.

В неприводимых решетках каждая грань параллелепипеда $\Pi\left(\gamma_{1}, \ldots, \gamma_{t}\right)$ содержит ровно одну точку из минимальной системы $S=\left(\gamma_{1}, \ldots, \gamma_{t}\right)$, поэтому ранг $t$ системы $S$ всегда не превосходит размерности $s$.

В двумерном случае, излагая на геометрическом языке классическую теорию цепных дробей, Вороной доказал (см. [4]), что минимальная система из двух векторов $\left\{\gamma_{a}, \gamma_{b}\right\}$ двумерной решетки $\Gamma$ всегда является базисом, а матрица $M\left(\gamma_{a}, \gamma_{b}\right)$ действием группы $G_{2}$ приводится к виду

$$
\left(\begin{array}{cc}
1 & -b_{1} \\
a_{2} & 1
\end{array}\right), \quad a_{2}, b_{1} \in[0,1] .
$$

Очевидно, что верно и обратное: пара векторов $\left(\gamma_{a}, \gamma_{b}\right)$, для которых матрица $M\left(\gamma_{a}, \gamma_{b}\right)$ эквивалентна матрице (2.1), образует минимальную систему в решетке $\Gamma=\left\langle\gamma_{a}, \gamma_{b}\right\rangle$. Базисы двумерных решеток, являющиеся минимальными системами, будем называть базисами Вороного.

В трехмерном случае векторы минимальной системы, состоящей из трех элементов, будем обозначать $\gamma_{a}, \gamma_{b}, \gamma_{c}$ и записывать в виде (1.2). Минимальной системе $S=\left(\gamma_{a}, \gamma_{b}, \gamma_{c}\right)$ будем 
ставить в соответствие матрицу

$$
M(S)=M\left(\gamma_{a}, \gamma_{b}, \gamma_{c}\right)=\left(\begin{array}{ccc} 
\pm a_{1} & \pm b_{1} & \pm c_{1} \\
\pm a_{2} & \pm b_{2} & \pm c_{2} \\
\pm a_{3} & \pm b_{3} & \pm c_{3}
\end{array}\right)
$$

записывая в столбцы координаты векторов системы.

Минимальную систему $\left(\gamma_{a}, \gamma_{b}, \gamma_{c}\right)$ будем называть вырожденной, если $\operatorname{det} M\left(\gamma_{a}, \gamma_{b}, \gamma_{c}\right)=0$; в противном случае систему будем называть невырожденной.

Группа $G_{3}$ сохраняет локальную структуру решетки. Матрицы, которые переводятся друг в друга действием группы $G_{3}$, будем называть эквивалентными.

Множество матриц минимальных систем, состоящих из трех векторов, под действием группы $G_{3}$ распадается на три орбиты. Каждая грань экстремального параллелепипеда $\Pi\left(\gamma_{a}, \gamma_{b}, \gamma_{c}\right)$ содержит ровно один из узлов $\pm \gamma_{a}, \pm \gamma_{b}, \pm \gamma_{c}$. Поэтому оси координат можно определить так, чтобы в строках матрицы $M\left(\gamma_{a}, \gamma_{b}, \gamma_{c}\right)$ наибольшие по модулю элементы были положительны и стояли на главной диагонали. За счет выбора масштаба на осях можно добиться того, чтобы диагональные элементы матрицы $M\left(\gamma_{a}, \gamma_{b}, \gamma_{c}\right)$ были равны 1. Таким образом, ситуация сводится к анализу минимальных систем с матрицей стандартного вида

$$
\left(\begin{array}{ccc}
1 & \pm b_{1} & \pm c_{1} \\
\pm a_{2} & 1 & \pm c_{2} \\
\pm a_{3} & \pm b_{3} & 1
\end{array}\right), \quad a_{2}, a_{3}, b_{1}, b_{3}, c_{1}, c_{2} \in[0,1] .
$$

После сделанной нормировки экстремальный параллелепипед $\Pi\left(\gamma_{a}, \gamma_{b}, \gamma_{c}\right)$ становится единичным кубом $\Pi:=(-1,1)^{3}$. Отметим, что для неприводимых решеток коэффициенты матрицы (2.3) удовлетворяют условиям $a_{2}, a_{3}, b_{1}, b_{3}, c_{1}, c_{2} \in(0,1)$.

Два вектора из одной минимальной системы не могут иметь равные (или противоположные знаки), так как иначе их разность (или сумма) нарушает минимальность данной системы. Это приводит к 24 возможным расстановкам знаков в матрице (2.3) (см. табл. 1).

\section{Таблица 1}

\begin{tabular}{ccc}
1 & $(1, \mathrm{II})$ \\
\hline+ & - & + \\
+ & + & + \\
- & + & +
\end{tabular}

\begin{tabular}{ccc}
2 & $(2, \mathrm{II})$ \\
\hline+ & + & + \\
- & + & + \\
+ & - & +
\end{tabular}

\begin{tabular}{ccc}
3 & $(3, \mathrm{II})$ \\
\hline+ & + & - \\
+ & + & + \\
+ & - & +
\end{tabular}

\begin{tabular}{ccc}
4 & $(4, \mathrm{I})$ \\
\hline+ & + & - \\
- & + & + \\
+ & - & +
\end{tabular}

\begin{tabular}{ccc}
$5(5, \mathrm{I})$ \\
\hline+ & - & + \\
+ & + & - \\
- & + & +
\end{tabular}

\begin{tabular}{ccc}
6 & $(6$, III $)$ \\
\hline+ & - & - \\
- & + & - \\
- & - & +
\end{tabular}

\begin{tabular}{ccc}
7 & $(1, \mathrm{II})$ \\
\hline+ & + & - \\
- & + & + \\
+ & + & +
\end{tabular}

\begin{tabular}{ccc}
8 & $(2, \mathrm{II})$ \\
\hline+ & - & + \\
+ & + & - \\
+ & + & +
\end{tabular}

\begin{tabular}{ccc}
9 & $(3, \mathrm{II})$ \\
\hline+ & + & + \\
+ & + & - \\
- & + & +
\end{tabular}

\begin{tabular}{ccc}
10 & $(4$, & $\mathrm{I})$ \\
\hline+ & - & - \\
+ & + & - \\
+ & + & +
\end{tabular}

\begin{tabular}{ccc}
11 & $(5, \mathrm{I})$ \\
\hline+ & + & + \\
- & + & + \\
- & - & +
\end{tabular}

\begin{tabular}{ccc}
12 & $(6$, III $)$ \\
\hline+ & + & - \\
+ & + & + \\
- & + & +
\end{tabular}

$13(1, \mathrm{II})$

\begin{tabular}{ccc}
14 & $(2, \mathrm{II})$ \\
\hline+ & + & - \\
- & + & - \\
- & + & +
\end{tabular}

\begin{tabular}{ccc}
15 & $(3, \mathrm{II})$ \\
\hline+ & - & + \\
- & + & + \\
- & - & +
\end{tabular}

\begin{tabular}{ccc}
16 & $(4, \mathrm{I})$ \\
\hline+ & + & + \\
- & + & - \\
- & + & +
\end{tabular}

\begin{tabular}{ccc}
17 & $(5, \mathrm{I})$ \\
\hline+ & - & - \\
+ & + & + \\
+ & - & +
\end{tabular}

\begin{tabular}{ccc}
18 & $(6$, III $)$ \\
\hline+ & + & + \\
+ & + & - \\
+ & - & +
\end{tabular}

\begin{tabular}{ccc}
19 & $(1$, & II $)$ \\
\hline+ & - & - \\
+ & + & - \\
+ & - & +
\end{tabular}

$$
\frac{20(2, \mathrm{II})}{+-}
$$$$
\begin{array}{lll}
21 & (3, \mathrm{II}) \\
\hline+ & - & - \\
- & + & - \\
+ & + & +
\end{array}
$$

\begin{tabular}{ccc}
22 & $(4, \mathrm{I})$ \\
\hline+ & - & + \\
+ & + & + \\
- & - & +
\end{tabular}

\begin{tabular}{ccc}
23 & $(5, \mathrm{I})$ \\
\hline+ & + & - \\
- & + & - \\
+ & + & +
\end{tabular}

\begin{tabular}{ccc}
24 & $(6$, III $)$ \\
\hline+ & - & + \\
- & + & + \\
+ & + & +
\end{tabular}

Вслед за номером в скобках арабскими цифрами указан номер эквивалентной сигнатуры из табл. 2 , а римскими - тип минимальной тройки (см. определения ниже). 
С помощью изменений знаков в строках и столбцах 24 варианта сводятся к следующим 6 (см. табл. 2).

Таблица 2. Нормальные сигнатуры и соответствующие им типы минимальных троек

\begin{tabular}{|c|c|c|c|c|c|c|c|c|c|c|c|c|c|c|c|c|c|}
\hline \multicolumn{3}{|c|}{1 (II) } & \multicolumn{3}{|c|}{$2(\mathrm{II})$} & \multicolumn{3}{|c|}{$3(\mathrm{II})$} & \multicolumn{3}{|c|}{$4(\mathrm{I})$} & \multicolumn{3}{|c|}{$5(\mathrm{I})$} & \multicolumn{3}{|c|}{$6(\mathrm{III})$} \\
\hline+ & - & + & + & + & + & + & + & - & + & + & - & + & - & + & + & - & - \\
\hline+ & + & + & - & + & + & + & + & + & - & + & + & + & + & - & - & + & - \\
\hline- & + & + & + & - & + & + & - & + & + & - & + & - & + & + & - & - & + \\
\hline
\end{tabular}

За счет перестановки строк и столбцов, сохраняющих диагональное преобладание элементов матрицы, число возможных конфигураций уменьшается до 3, т.е. множество всех матриц минимальных систем под действием группы $G_{3}$ распадается на 3 класса эквивалентности с представителями вида

$$
\left(\begin{array}{rrr}
1 & b_{1} & -c_{1} \\
-a_{2} & 1 & c_{2} \\
a_{3} & -b_{3} & 1
\end{array}\right), \quad\left(\begin{array}{rrr}
1 & b_{1} & c_{1} \\
-a_{2} & 1 & c_{2} \\
a_{3} & -b_{3} & 1
\end{array}\right), \quad\left(\begin{array}{rrr}
1 & -b_{1} & -c_{1} \\
-a_{2} & 1 & -c_{2} \\
-a_{3} & -b_{3} & 1
\end{array}\right)
$$

где $a_{i}, b_{i}, c_{i} \in[0,1]$.

Этим трем матрицам соответствует три типа минимальных троек, которые можно считать трехмерными аналогами базисов Вороного. Точное описание минимальных троек дает следующий результат Минковского.

Теорема 1 (Минковский). Пусть $S=\left(\gamma_{a}, \gamma_{b}, \gamma_{c}\right)$ - минимальная система решетки $Г$. Если система $S$ невырожденная, то тройка векторов $\left(\gamma_{a}, \gamma_{b}, \gamma_{c}\right)$ является базисом решетки Г, а матрица $M\left(\gamma_{a}, \gamma_{b}, \gamma_{c}\right)$ эквивалентна одной из двух канонических форм

$$
\begin{array}{ccc}
M_{1}=\left(\begin{array}{rrr}
1 & b_{1} & -c_{1} \\
-a_{2} & 1 & c_{2} \\
a_{3} & -b_{3} & 1
\end{array}\right), & a_{2}, a_{3}, b_{2}, b_{3}, c_{1}, c_{2} \in[0,1], \\
M_{2}=\left(\begin{array}{rrr}
1 & b_{1} & c_{1} \\
-a_{2} & 1 & c_{2} \\
a_{3} & -b_{3} & 1
\end{array}\right), & c_{2}, a_{3}, b_{2}, b_{3}, c_{1}, c_{2} \in[0,1], \\
\end{array}
$$

Если же система $S$ вырожденная, то для некоторой комбинации знаков $\gamma_{a} \pm \gamma_{b} \pm \gamma_{c}=0$, а матрица $M\left(\gamma_{a}, \gamma_{b}, \gamma_{c}\right)$ действием группь $G_{3}$ приводится к виду

$$
M_{3}=\left(\begin{array}{ccc}
1 & -b_{1} & -c_{1} \\
-a_{2} & 1 & -c_{2} \\
-a_{3} & -b_{3} & 1
\end{array}\right), \quad b_{1}+c_{1}=1, \quad a_{2}, a_{3}, b_{2}, b_{3}, c_{1}, c_{2} \in[0,1], \quad a_{3}+b_{3}=1
$$

Верно и обратное: система из трех векторов $\left(\gamma_{a}, \gamma_{b}, \gamma_{c}\right)$, матрица которой эквивалентна одной из матрии вида (2.4), (2.5), является минимальной системой полной решетки $\Gamma=\left\langle\gamma_{a}, \gamma_{b}, \gamma_{c}\right\rangle ;$ система векторов $\left(\gamma_{a}, \gamma_{b}, \gamma_{c}\right)$ с матрищей вида (2.6) является минимальной системой решетки (ранга 2) $\Gamma=\left\langle\gamma_{a}, \gamma_{b}, \gamma_{c}\right\rangle=\left\langle\gamma_{a}, \gamma_{b}\right\rangle$.

Минковским эта теорема сформулирована без доказательства (см. [5], [6]). Подробное доказательство может быть найдено в [11, Art. 109-110] (см. также [18], [22]-[24]).

В соответствии с теоремой 1 минимальные системы, матрицы которых эквивалентны матрицам (2.4), (2.5), будем называть базисами Минковского I и II типа соответственно, а минимальные системы вида (2.6) - вырожденными тройками Минковского или тройками III muna. 
ЗАмЕчаниЕ 1. Минковским неравенства на элементы матриц (2.4)-(2.5) записывались в более симметричной форме. В матрице $M_{1}$

$$
c_{1} \leqslant b_{1} \text { или } a_{2} \leqslant c_{2} \text { или } b_{3} \leqslant a_{3} ;
$$

в матрице $M_{2} a_{2}+c_{2} \geqslant 1$ и

$$
c_{1} \leqslant b_{1} \quad \text { или } a_{3} \leqslant b_{3} .
$$

Переход к более простым, хотя менее симметричным условиям, в (2.4) и (2.5) может быть сделан за счет эквивалентных преобразований: в матрице (2.4) достаточно сделать циклическую перестановку строк и столбцов, а в матрице (2.5) - поменять местами столбцы 1,3 и строки 1, 3 (сигнатуры, имеющие в табл. 1 номера 2 и 8, эквивалентны друг другу).

\section{3. Минимальные системы в приводимых решетках}

Определение минимальных систем, данное ранее для неприводимых решеток, можно непосредственно перенести и на произвольные решетки. Однако при таком подходе возникает ряд трудностей, которые приводят к излишнему усложнению ситуации. В некоторых решетках будут существовать минимальные системы более чем из 3 векторов. Выпуклая оболочка векторов в таких системах может содержать точки решетки на своих ребрах и внутри граней. Индекс минимальной системы может достигать значений 3 и 4. (Например, подобная ситуация возникает на решетке $\mathbb{Z}^{3}$, когда на поверхности куба $[-1,1]^{3}$ находится минимальная система из 13 векторов, некоторые из которых лежат на серединах ребер или в центрах граней.) При этом классификация минимальных систем становится очень громоздкой. Трехмерный аналог теоремы Валена перестает выполняться, пример с тройкой векторов $\gamma_{a}=(-1,1,1)$, $\gamma_{b}=(1,-1,1), \gamma_{c}=(1,1,-1)$ из решетки $\mathbb{Z}^{3}$ показывает, что нельзя доказать ничего лучше тривиальной оценки (1.4).

Ситуация становится более естественной, если к определению минимальных систем добавить дополнительные условия.

Пусть имеется тройка линейно независимых векторов $\gamma_{a}, \gamma_{b}, \gamma_{c} \in \Gamma$. Октаэдр с вершинами $\pm \gamma_{a}, \pm \gamma_{b}, \pm \gamma_{c}$ назовем nycmым, если он не содержит (строго) внутри себя точек решетки $\Gamma$, отличных от начала координат. Пустой октаэдр, который не содержит на своей поверхности точек решетки $\Gamma$, отличных от $\pm \gamma_{a}, \pm \gamma_{b}, \pm \gamma_{c}$, будем называть примитивным.

Для произвольной решетки $\Gamma \in \mathcal{L}_{3}(\mathbb{R})$ систему векторов $\left(\gamma_{1}, \ldots, \gamma_{t}\right)$ назовем минималъной, если выполняются следующие условия:

1) система состоит не более чем из трех векторов;

2) параллелепипед $\Pi\left(\gamma_{1}, \ldots, \gamma_{t}\right)$ не содержит точек решетки $Г$, отличных от начала координат;

3) если $t=3$ и система $\left(\gamma_{1}, \ldots, \gamma_{t}\right)$ невырождена, то октаэдр с вершинами $\pm \gamma_{1}, \pm \gamma_{2}, \pm \gamma_{3}$ является примитивным.

Для решеток общего положения первое и последнее требования выполняются автоматически, поэтому новое определение не противоречит данному ранее.

Более общая ситуация, когда тройка векторов удовлетворяет двум первым требованиям, но не удовлетворяет последнему, сводится к анализу минимальных систем с помощью теоремы 3 (см. ниже).

При анализе минимальных систем будем предполагать, что в каждой строке есть ненулевой элемент, поскольку в противном случае задача становится плоской и полное описание сводится к исследованию базисов Вороного (2.1). В классах эквивалентности (как и в случае неприводимых решеток) представители обычно будут выбираться так, чтобы в каждой строке наибольший по модулю элемент был равен 1. При этом по определению минимальной системы в каждом столбце матрицы $M\left(\gamma_{a}, \gamma_{b}, \gamma_{c}\right)$ также должен быть элемент, равный по модулю 1. 
Таким образом, векторы минимальной системы всегда будут находиться на поверхности куба $\Pi=(-1,1)^{3}$.

При переходе от неприводимых решеток к приводимым строение множества минимальных систем существенно усложняется. Чтобы подчеркнуть отличия, сравним свойства минимальных систем в неприводимых и приводимых решетках.

\section{Свойства минимальных систем в неприводимых решетках}

$1^{\circ}$. Два вектора из одной минимальной системы не могут лежать в одном октанте (октанты предполагаются замкнутыми, противоположные октанты отождествляются). Другими словами, два вектора из минимальной системы не могут иметь равные или противоположные сигнатуры.

$2^{\circ}$. Если $\left(\gamma_{a}, \gamma_{b}, \gamma_{c}\right)$ - минимальная система решетки $\Gamma$, то каждая грань экстремального параллелепипеда $\Pi\left(\gamma_{a}, \gamma_{b}, \gamma_{c}\right)$ содержит ровно одну точку решетки. Матрица $M\left(\gamma_{a}, \gamma_{b}, \gamma_{c}\right)$ с помощью элементарных преобразований может быть приведена к стандартному виду (к матрице с диагональным преобладанием) (2.3), а векторы $\pm \gamma_{a}, \pm \gamma_{b}, \pm \gamma_{c}$ имеют попарно различные сигнатуры.

$3^{\circ}$. Минимальные тройки удовлетворяют теореме Минковского 1 , т.е. матрица $M\left(\gamma_{a}, \gamma_{b}, \gamma_{c}\right)$ эквивалентна одной из матриц (2.4)-(2.6) с соответствующими ограничениями на элементы. В частности, индекс минимальной тройки может быть равен 0 или 1.

$4^{\circ}$. Каждый вектор минимальной системы является локальным минимумом решетки.

\section{Свойства минимальных систем в приводимых решетках}

$1^{\circ}$. Два вектора из одной минимальной системы могут лежать в одном октанте. Более того, существуют минимальные системы из трех элементов, обладающие следущим свойством: при любом доопределении знаков у нулевых координат векторы системы будут содержаться в объединении двух октантов. Такие системы в дальнейшем будем называть лежащими в двух октантах. (См. примеры в теореме 6.)

$2^{\circ}$. Два вектора из минимальной системы $\left(\gamma_{a}, \gamma_{b}, \gamma_{c}\right)$ могут находиться строго внутри одной грани экстремального параллелепипеда $\Pi\left(\gamma_{a}, \gamma_{b}, \gamma_{c}\right)$. Соответственно матрица $M\left(\gamma_{a}, \gamma_{b}, \gamma_{c}\right)$ может быть не эквивалентна матрице (2.3). Такие системы будем называть системами без диагонального преобладания. (См. примеры в теореме 7.)

$3^{\circ}$. Если векторы минимальной системы $S=\left(\gamma_{a}, \gamma_{b}, \gamma_{c}\right)$ лежат в трех попарно различных октантах и матрица $M\left(\gamma_{a}, \gamma_{b}, \gamma_{c}\right)$ действием группы $G_{3}$ приводится к стандартному виду (2.3), то данная система все равно может не удовлетворять теореме Минковского 1: тройки со знаками как в матрицах $(2.4),(2.5)$, могут не быть базисами и иметь индекс 0 или 2 ; тройка $\left(\gamma_{a}, \gamma_{b}, \gamma_{c}\right)$ со знаками, как в матрице $(2.6)$, может быть базисом решетки, а может образовывать вырожденную систему, для которой $\gamma_{a}+\gamma_{b}+\gamma_{c} \neq 0$. (См. примеры в теоремах 8-10.)

$4^{\circ}$. Вектор минимальной системы может не быть локальным минимумом решетки.

Минимальную систему, в которой каждый вектор является минимумом решетки, будем называть вполне минимальной. Для неприводимых решеток понятия минимальной и вполне минимальной систем равносильны. Для приводимых решеток класс вполне минимальных систем у́же. Например, на решетке $\mathbb{Z}^{3}$ система из трех векторов $(0,0,1),(0,1,1),(1,1,1)$ является минимальной, но не вполне минимальной.

В теореме 11 будет доказано, что для вполне минимальных систем в произвольных решетках остается справедливым неравенство (1.3) из трехмерного аналога теоремы Валена. На множестве всех минимальных систем будет выполняться ослабленный вариант неравенства (1.3), который, однако, точнее тривиальной оценки (1.4).

Минимальные тройки, матрицы которых приводятся к виду (2.4)-(2.6), будем называть минимальными системами Минковского (они соответственно состоят из базисов Минковского 
I $и$ II типов и вырожденных минимальных систем Минковского), все остальные минимальные тройки будем называть исключителъными. В соответствии с вышесказанным исключительные минимальные тройки можно поделить на следующие три категории (с непустыми пересечениями):

- тройки, лежащие в двух октантах;

- тройки без диагонального преобладания;

- исключительные тройки стандартного вида (2.3).

Внутри каждой категории тройки будем отличать по их индексу, который по теореме 2 (см. ниже) может принимать значения от 0 до 2.

Далее для каждого типа троек будет получено описание, аналогичное описанию минимальных систем Минковского в теореме 1. Всего получится 12 типов минимальных троек. Описание 9 типов исключительных троек будет дано в теоремах 6-10 ниже.

\section{4. Основной инструментарий: теоремы Минковского и их обобщения}

При анализе минимальных систем понадобится ряд вспомогательных утверждений о свойствах трехмерных решеток.

ТЕОРема 2 (Минковский). Пусть имеется примитивный октаэдр с вершинами $\pm \gamma_{a}$, $\pm \gamma_{b}, \pm \gamma_{c} \in \Gamma$. Тогда либо набор $\left(\gamma_{a}, \gamma_{b}, \gamma_{c}\right)$ является базисом решетки Г, либо $\left(\gamma_{a}, \gamma_{b}, \gamma_{c}\right)-$ система индекса 2 в решетке $Г и \Gamma=\left\langle\gamma_{a}, \gamma_{b},\left(\gamma_{a}+\gamma_{b}+\gamma_{c}\right) / 2\right\rangle$.

ДокАЗАтЕльство см. в [25, с. 97-101] или [11, Art. 161].

Как отмечалось выше, аналогичное утверждение для пустых, но не примитивных октаэдров, позволяет описывать конфигурации точек решетки на поверхности произвольного экстремального параллелепипеда.

ТЕОрема 3. Пусть октаэдр с вершинами $\pm \gamma_{a}, \pm \gamma_{b}, \pm \gamma_{c} \in \Gamma$ является пустым, но не примитивным. Тогда выполнен один из следующих трех вариантов (см. рис. 1):

1) $I\left(\gamma_{a}, \gamma_{b}, \gamma_{c}\right)=2$ и базисом решетки Г является один из наборов

$$
\left(\gamma_{a}, \gamma_{b}, \frac{\gamma_{a} \pm \gamma_{b}}{2}\right), \quad\left(\gamma_{b}, \gamma_{c}, \frac{\gamma_{b} \pm \gamma_{c}}{2}\right), \quad\left(\gamma_{c}, \gamma_{a}, \frac{\gamma_{c} \pm \gamma_{a}}{2}\right)
$$

2) $I\left(\gamma_{a}, \gamma_{b}, \gamma_{c}\right)=3$ и базисом решетки Г является один из наборов

$$
\left(\gamma_{a}, \gamma_{b}, \frac{\gamma_{a} \pm \gamma_{b} \pm \gamma_{c}}{3}\right)
$$

3) $I\left(\gamma_{a}, \gamma_{b}, \gamma_{c}\right)=4$ и базисом решетки Г является один из наборов

$$
\begin{gathered}
\left(\gamma_{a}, \gamma_{b}, \frac{2 \gamma_{a} \pm \gamma_{b} \pm \gamma_{c}}{4}\right), \quad\left(\gamma_{b}, \gamma_{c}, \frac{2 \gamma_{b} \pm \gamma_{c} \pm \gamma_{a}}{4}\right) \\
\left(\gamma_{c}, \gamma_{a}, \frac{2 \gamma_{c} \pm \gamma_{a} \pm \gamma_{b}}{4}\right), \quad\left(\frac{\gamma_{a}+\gamma_{b}}{2}, \frac{\gamma_{b}+\gamma_{c}}{2}, \frac{\gamma_{a}+\gamma_{a}}{2}\right) .
\end{gathered}
$$

Для доказательства теоремы 3 применим следующее утверждение (см. [26, лемма 2]).

Лемма 1. Пусть $\alpha_{1}, \alpha_{2}, \alpha_{3}$ - вещественные числа $u\left\|\alpha_{1}\right\| \leqslant\left\|\alpha_{2}\right\| \leqslant\left\|\alpha_{3}\right\|$. Тогда

$$
\min \left\{\left\|\alpha_{1}\right\|+\left\|\alpha_{2}\right\|+\left\|\alpha_{3}\right\|,\left\|2 \alpha_{1}\right\|+\left\|2 \alpha_{2}\right\|+\left\|2 \alpha_{3}\right\|\right\} \leqslant 1 .
$$

Eсли

$$
\min \left\{\left\|\alpha_{1}\right\|+\left\|\alpha_{2}\right\|+\left\|\alpha_{3}\right\|,\left\|2 \alpha_{1}\right\|+\left\|2 \alpha_{2}\right\|+\left\|2 \alpha_{3}\right\|\right\}=1
$$




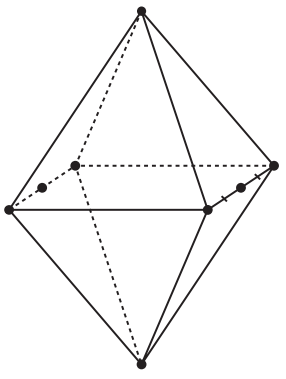

$I=2$

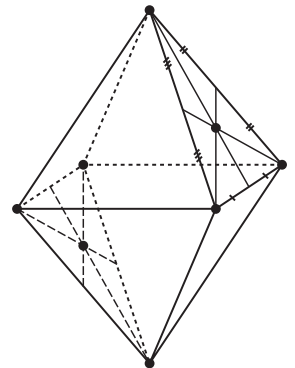

$I=3$

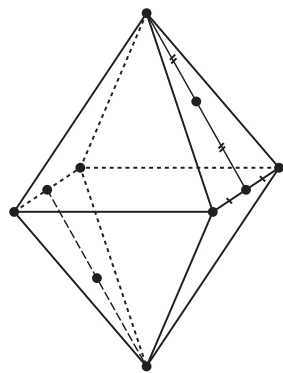

$I=4$

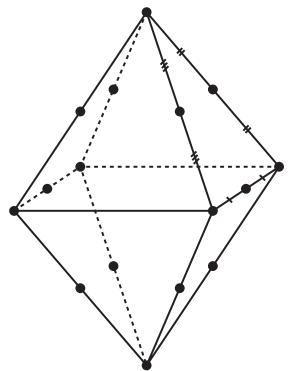

$I=4$

Рис. 1. Возможные конфигурации точек решетки на поверхности пустого, но не примитивного октаэдра

mo

$$
\left\|3 \alpha_{1}\right\|+\left\|3 \alpha_{2}\right\|+\left\|3 \alpha_{3}\right\| \leqslant 1
$$

и равенство возможно только в случае, когда $\left\|\alpha_{1}\right\|=1 / 4,\left\|\alpha_{2}\right\|=1 / 4,\left\|\alpha_{3}\right\|=1 / 2$.

ДоКАЗАТЕЛЬСТВО ТЕОРЕМЫ 3. Поскольку данный октаэдр не является примитивным, $I\left(\gamma_{a}, \gamma_{b}, \gamma_{c}\right) \geqslant 2$. Поэтому найдется ненулевой узел решетки

$$
\gamma=k_{a} \gamma_{a}+k_{b} \gamma_{b}+k_{c} \gamma_{c}
$$

для которого $k_{a}, k_{b}, k_{c} \in(-1 / 2,1 / 2]$. Будем предполагать, что порядок векторов $\gamma_{a}, \gamma_{b}, \gamma_{c}$ выбран так, что $\left\|k_{a}\right\| \leqslant\left\|k_{b}\right\| \leqslant\left\|k_{c}\right\|$. Приводя коэффициенты $k_{a}, k_{b}, k_{c}$ к общему знаменателю, запишем их в виде

$$
k_{a}=\frac{m_{a}}{n}, \quad k_{b}=\frac{m_{b}}{n}, \quad k_{c}=\frac{m_{c}}{n}, \quad n>1 .
$$

Если можно найти несколько таких векторов $\gamma$, то выберем среди них тот, для которого значение $n$ принимает наибольшее значение.

Вектор

$$
\gamma_{l}=\left\|l k_{a}\right\| \gamma_{a}+\left\|l k_{b}\right\| \gamma_{b}+\left\|l k_{c}\right\| \gamma_{c}, \quad l=1,2, \ldots,
$$

не лежит внутри октаэдра с вершинами $\pm \gamma_{a}, \pm \gamma_{b}, \pm \gamma_{c}$ тогда и только тогда, когда

$$
\left\|l k_{a}\right\|+\left\|l k_{b}\right\|+\left\|l k_{c}\right\| \geqslant 1 \text {. }
$$

По лемме 1 вектор $\left(\left\|k_{a}\right\|,\left\|k_{b}\right\|,\left\|k_{c}\right\|\right)$ должен совпадать с одним из наборов

$$
\left(0, \frac{1}{2}, \frac{1}{2}\right), \quad\left(\frac{1}{2}, \frac{1}{2}, \frac{1}{2}\right), \quad\left(\frac{1}{3}, \frac{1}{3}, \frac{1}{3}\right), \quad\left(\frac{1}{4}, \frac{1}{4}, \frac{1}{2}\right)
$$

Если предположить, что существует точка решетки

$$
\gamma^{\prime}=k_{a}^{\prime} \gamma_{a}+k_{b}^{\prime} \gamma_{b}+k_{c}^{\prime} \gamma_{c}
$$

такая, что $k_{a}^{\prime}, k_{b}^{\prime}, k_{c}^{\prime} \in(-1 / 2,1 / 2]$ и

$$
\left(k_{a}^{\prime}, k_{b}^{\prime}, k_{c}^{\prime}\right) \not \equiv\left(l k_{a}, l k_{b}, l k_{c}\right)(\bmod 1), \quad l=1,2, \ldots,
$$

то набор $\left(\left\|k_{a}^{\prime}\right\|,\left\|k_{b}^{\prime}\right\|,\left\|k_{c}^{\prime}\right\|\right)$ с точностью до перестановки также должен быть одним из наборов (4.1). Если набор $\left(\left\|k_{a}^{\prime}\right\|,\left\|k_{b}^{\prime}\right\|,\left\|k_{c}^{\prime}\right\|\right)$ не совпадает с одним из наборов $(1 / 2,0,1 / 2)$, $(1 / 2,1 / 2,0)$, то, рассматривая линейные комбинации векторов $\gamma, \gamma^{\prime}, \gamma_{a}, \gamma_{b}, \gamma_{c}$, можно получить 
вектор с коэффициентами, которые (вместе со всеми своими перестановками) не содержатся в списке (4.1). Полученное противоречие показывает, что либо $\Gamma=\left\langle\gamma, \gamma_{b}, \gamma_{c}\right\rangle$, либо

$$
\Gamma=\left\langle\frac{\gamma_{a}+\gamma_{b}}{2}, \frac{\gamma_{a}+\gamma_{c}}{2}, \frac{\gamma_{b}+\gamma_{c}}{2}\right\rangle
$$

Набор коэффициентов $(1 / 2,1 / 2,1 / 2)$ является лишним, так как соответствующий ему октаэдр является примитивным. Оставшиеся варианты приводят к конфигурациям, описанным в условии теоремы.

ТеОремА 4 (Минковский). Пусть $\Omega$ - выпуклое иентралъно-симметричное тело с иентром симметрии в начале координат. Предположим, что вериинъ $\pm \gamma_{a}, \pm \gamma_{b}, \pm \gamma_{c} \in \Gamma$ nримитивного октаэдра лежат на поверхности $\Omega$.

1) Eсли $\left|\operatorname{det}\left(\gamma_{a}, \gamma_{b}, \gamma_{c}\right)\right|=\operatorname{det} \Gamma$, то внутренность области $\Omega$ не содержит точек решетки Г, отличных от начала координат тогда и только тогда, когда внутри $\Omega$ не лежат линейные комбинации узлов $\gamma_{a}, \gamma_{b}, \gamma_{c}$ с коэфбициентами из следующих 22 наборов:

$$
\begin{gathered}
(1, \pm 1,0), \quad(1,0, \pm 1), \quad(0,1, \pm 1), \quad(1, \pm 1, \pm 1), \\
(2, \pm 1, \pm 1), \quad( \pm 1,2, \pm 1), \quad( \pm 1, \pm 1,2) .
\end{gathered}
$$

2) Если $\left|\operatorname{det}\left(\gamma_{a}, \gamma_{b}, \gamma_{c}\right)\right|=2 \operatorname{det} \Gamma$, то внутренность области $\Omega$ не содержит точек решетки Г, отличных от начала координат тогда и только тогда, когда внутри $\Omega$ не лежат линейные комбинации узлов $\gamma_{a}, \gamma_{b}, \gamma_{c}$ с коэфбициентами

$$
\left(\frac{1}{2}, \pm \frac{1}{2}, \pm \frac{1}{2}\right) \text {. }
$$

ДокАЗАТЕЛьСтво см. в [25, с. 101-104], [27, с. 12-14] или [11, Art. 162].

Теорему 4 можно дополнить следующим утверждением, которое будет применяться к анализу вырожденных троек.

ТеОРема 5. Пусть $\Omega$ - выпуклое центрально-симметричное тело с центром симметрии в начале координат. Предположим, что узлы системы $\left(\gamma_{a}, \gamma_{b}, \gamma_{c}\right)$ решетки Г лежат на поверхности $\Omega$ u $\operatorname{det}\left(\gamma_{a}, \gamma_{b}, \gamma_{c}\right)=0$.

1) Внутренность области $\Omega$ не содержит точек решетки $\Gamma$, отличных от начала координат тогда и только тогда, когда внутри $\Omega$ не лежат ненулевые линейные комбинации узлов $\gamma_{a}, \gamma_{b}, \gamma_{c}$ с коэфбициентами из следующего списка:

$$
(1, \pm 1,0), \quad(1,0, \pm 1), \quad(0,1, \pm 1), \quad(1, \pm 1, \pm 1) .
$$

2) Если внутренность области $\Omega$ не содержит точек решетки $\Gamma$, отличньх от начала координат, то среди линейных комбинаций векторов $\gamma_{a}, \gamma_{b}, \gamma_{c} c$ коэфбициентами из следующего списка:

$$
(1, \pm 1, \pm 1), \quad(2, \pm 1, \pm 1), \quad( \pm 1,2, \pm 1), \quad( \pm 1, \pm 1,2)
$$

будет присутствовать нулевой вектор.

ДокАЗАТЕЛЬСтво. 1) Необходимость указанных условий очевидна. Докажем их достаточность. Проведем через узлы $\gamma_{a}, \gamma_{b}, \gamma_{c}$ плоскость $\pi$ и положим $\Gamma^{\prime}=\Gamma \cap \pi$. Пусть $M-$ выпуклая оболочка точек $\pm \gamma_{a}, \pm \gamma_{b}, \pm \gamma_{c}$. Многоугольник $M$ может содержать ненулевые узлы решетки $\Gamma^{\prime}$ только на своей границе. Если таких точек $n$, то площадь $M$ равна $(n / 2) \operatorname{det} \Gamma^{\prime}$. По теореме Минковского о выпуклом теле эта площадь не может быть больше, чем $4 \operatorname{det} \Gamma^{\prime}$, т.е. $n \leqslant 8$. Следовательно, $M$ - либо шестиугольник, либо параллелограмм, у которого середины сторон 
также являются узлами решетки $\Gamma^{\prime}$. Будем считать, что $\gamma_{a}, \gamma_{b}, \gamma_{c}$ являются последовательными точками решетки на периметре $M$ (этого всегда можно добиться за счет изменения ориентации векторов). Чтобы область $\Omega^{\prime}=\Omega \cap \pi$ не содержала точек решетки $\Gamma$, в случае шестиугольника достаточно выполнения условия

$$
\gamma_{a}+\gamma_{b}, \gamma_{b}+\gamma_{c}, \gamma_{c}-\gamma_{a} \notin \Omega^{\prime}
$$

В случае параллелограмма достаточно, чтобы

$$
\gamma_{a}+\gamma_{b}-\gamma_{c}, \gamma_{c}-\gamma_{a}, \gamma_{b}+\gamma_{c}-\gamma_{a} \notin \Omega^{\prime}
$$

Таким образом, в обоих случаях достаточно проверить линейные комбинации векторов $\gamma_{a}$, $\gamma_{b}, \gamma_{c}$ с коэффициентами (4.4).

2) Снова предположим, что $\gamma_{a}, \gamma_{b}, \gamma_{c}$ являются последовательными точками решетки на периметре выпуклой оболочки $M$ точек $\pm \gamma_{a}, \pm \gamma_{b}, \pm \gamma_{c}$. Если $M$ - шестиугольник, то $\gamma_{a}-$ $\gamma_{b}+\gamma_{c}=0$. Если $M$ - параллелограмм, то $\gamma_{a}-2 \gamma_{b}+\gamma_{c}=0$. В обоих случаях достаточно проверить линейные комбинации векторов $\gamma_{a}, \gamma_{b}, \gamma_{c}$ с коэффициентами (4.5).

\section{5. Классификация исключительных минимальных систем}

В теоремах 6-10 будет дано описание исключительных минимальных троек. Доказательства теорем будут проходить примерно по одной и той же схеме, позаимствованной из доказательства теоремы Минковского 1. Для невырожденных минимальных систем $S$ необходимые и достаточные условия на коэффициенты матрицы $M(S)$ будут находиться с помощью теоремы 4, а для вырожденных - из теоремы 5.

При расстановке знаков в матрицах минимальных систем будем предполагать, что нулевым координатам могут быть присвоены оба знака.

\section{1. Минимальные системы, лежащие в двух октантах.}

Теорема 6. Пусть $S=\left(\gamma_{a}, \gamma_{b}, \gamma_{c}\right)$ - такая минимальная система решетки Г, что при любой расстановке знаков векторы $\pm \gamma_{a}, \pm \gamma_{b}, \pm \gamma_{c}$ содержатся в оббединении двух октантов. Тогда если $S$ - вырожденная тройка, то матрища $M\left(\gamma_{a}, \gamma_{b}, \gamma_{c}\right)$ эквивалентна одной из двух матрии, (см. рис. 2)

$$
\begin{array}{ll}
M_{4}=\left(\begin{array}{ccc}
0 & 1 & 1 \\
1 & b_{2} & b_{2}-1 \\
1 & b_{3} & b_{3}-1
\end{array}\right), & b_{2}, b_{3} \in(0,1), \\
M_{5}=\left(\begin{array}{ccc}
0 & 1 & 1 \\
1 & 1 & -1 \\
\frac{1+c_{2}}{2} & 1 & -c_{3}
\end{array}\right), & c_{3} \in(0,1] .
\end{array}
$$

Если $S$ - невьрожденная тройка, то она является базисом решетки Г, а матрица $M\left(\gamma_{a}, \gamma_{b}, \gamma_{c}\right)$ эквивалентна матрице вида

$$
M_{6}=\left(\begin{array}{ccc}
0 & 1 & 1 \\
1 & b_{2} & -c_{2} \\
a_{3} & 1 & -c_{3}
\end{array}\right), \quad 2 a_{3} \leqslant c_{3} \quad \text { uли } a_{3} \leqslant c_{3}, c_{2}, b_{2}+c_{2} \leqslant 1 .
$$

Верно и обратное: система из трех векторов $\left(\gamma_{a}, \gamma_{b}, \gamma_{c}\right)$, матрица которой эквивалентна одной из матрии вида (5.1), (5.2), является минимальной системой решетки (ранга 2) $\Gamma=\left\langle\gamma_{a}, \gamma_{b}, \gamma_{c}\right\rangle=\left\langle\gamma_{b}, \gamma_{c}\right\rangle ;$ система векторов $\left(\gamma_{a}, \gamma_{b}, \gamma_{c}\right)$ с матрищей вида (5.3) является минимальной системой полной решетки $\Gamma=\left\langle\gamma_{a}, \gamma_{b}, \gamma_{c}\right\rangle$. Во всех случаях у векторов $\gamma_{a}, \gamma_{b}, \gamma_{c}$ нельзя выбрать ориентацию так, чтобы они попали в три попарно различных октанта. 


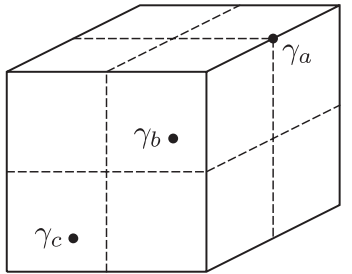

$M_{4}, \quad \gamma_{b}-\gamma_{c}=\gamma_{a}$

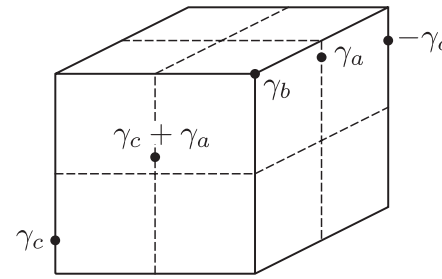

$M_{5}, \quad 2 \gamma_{a}=\gamma_{b}-\gamma_{c}$

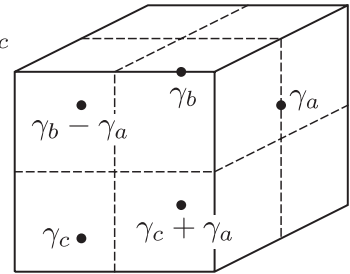

$M_{6}$

Рис. 2. Расположение точек минимальных систем с матрицами $M_{4}-M_{6}$

ДокАЗАТЕЛЬСтво. Если векторы $\gamma_{a}, \gamma_{b}, \gamma_{c}$ содержатся в двух октантах, то из них можно выбрать пару векторов, которые содержатся в одном октанте. Пусть это векторы $\gamma_{a}, \gamma_{b}$, и пусть они лежат октанте $\left\{\left(x_{1}, x_{2}, x_{3}\right): x_{1}, x_{2}, x_{3} \geqslant 0\right\}$. Их разность $\gamma_{a}-\gamma_{b}=\left(a_{1}-b_{1}, a_{2}-b_{2}\right.$, $\left.a_{3}-b_{3}\right)$ не нарушает минимальности данной системы $S$, если выполнено по крайней мере одно из равенств

$$
\left|a_{1}-b_{1}\right|=1, \quad\left|a_{2}-b_{2}\right|=1, \quad\left|a_{3}-b_{3}\right|=1 \text {. }
$$

Не ограничивая общности, будем считать, что $\left|a_{1}-b_{1}\right|=1$. Равенства $a_{1}-b_{1}=1$ и $a_{1}-b_{1}=-1$ эквивалентны (они могут быть переведены друг в друга действием группы $G_{3}$ ), поэтому достаточно ограничиться случаем, когда $a_{1}-b_{1}=-1$, т.е. $a_{1}=0, b_{1}=1$. Одна из координат вектора $\gamma_{a}$ должна быть равна 1 , поэтому можно считать, что $a_{2}=1$. При этом должно выполняться неравенство $a_{3}>0$, поскольку при $a_{3}=0$ вектор $\gamma_{a}$ будет лежать сразу в четыpex октантах, и при некоторой расстановке знаков векторы $\pm \gamma_{a}, \pm \gamma_{b}, \pm \gamma_{c}$ попадут в попарно различные октанты. Таким образом, достаточно ограничиться рассмотрением матриц вида

$$
\left(\begin{array}{ccc}
0 & 1 & \pm c_{1} \\
1 & b_{2} & \pm c_{2} \\
a_{3} & b_{3} & \pm c_{3}
\end{array}\right), \quad a_{3}>0 .
$$

Поскольку $a_{1}=0$, то можно вектору $\gamma_{a}$ приписать сигнатуру $(-++)$ и считать, что $\gamma_{a}$ и $\gamma_{b}$ лежат в различных октантах. По условию теоремы вектор $\gamma_{c}$ лежит в объединении этих октантов и, кроме того, он не может иметь неотрицательные координаты. Значит, $\gamma_{c}$ должен иметь вид $\gamma_{c}=\left(c_{1},-c_{2},-c_{3}\right)$. Кроме того, каждая строка матрицы $M\left(\gamma_{a}, \gamma_{b}, \gamma_{c}\right)$ должна содержать единичный по модулю элемент. Существуют лишь три матрицы, удовлетворяющие этим условиям:

$$
\begin{aligned}
& \left(\begin{array}{rrr}
0 & 1 & c_{1} \\
1 & b_{2} & -c_{2} \\
1 & b_{3} & -c_{3}
\end{array}\right), \\
& \left(\begin{array}{rrr}
0 & 1 & c_{1} \\
1 & b_{2} & -c_{2} \\
a_{3} & 1 & -c_{3}
\end{array}\right), \\
& \left(\begin{array}{ccr}
0 & 1 & c_{1} \\
1 & b_{2} & -c_{2} \\
a_{3} & b_{3} & -1
\end{array}\right) .
\end{aligned}
$$

Отметим, что элементы $a_{3}, b_{2}, b_{3}, c_{2}, c_{3}$ не могут быть нулевыми, так как в противном случае, поменяв знак у нулевого элемента, мы получим тройку векторов, лежащих в попарно различных октантах. Отсюда следует, что для всех трех матриц (5.4)-(5.6) вектор $\gamma_{a}+\gamma_{c}=$ $\left(c_{1}, \ldots\right)$ не будет нарушать минимальности данной системы $S$, только если $c_{1}=1$. 
Предположим сначала, что $S$ - вырожденная система. Рассматривая линейные комбинации векторов $\gamma_{a}, \gamma_{b}, \gamma_{c}$ с коэффициентами (4.5), получим, что для матрицы набора (5.4) в нуль может обращаться только вектор

$$
\gamma_{a}-\gamma_{b}+\gamma_{c}=\left(0,1-b_{2}-c_{2}, 1-b_{3}-c_{3}\right) .
$$

В этом случае $c_{2}=1-b_{2}, c_{3}=1-b_{3}$ и получаются тройки вида (5.1). Для матрицы $(5.5)$ нулевой может быть только комбинация

$$
2 \gamma_{a}-\gamma_{b}+\gamma_{c}=\left(0,2-b_{2}-c_{2}, 2 a_{3}-1-c_{3}\right)
$$

Если это действительно так, то должны выполняться равенства $b_{2}=c_{2}=1, a_{3}=\left(c_{3}+1\right) / 2$. Значит, матрица данной минимальной системы эквивалентна матрице (5.2). Для матрицы (5.6) нулевой может быть только комбинация

$$
2 \gamma_{a}-\gamma_{b}-\gamma_{c}=\left(0,2-b_{2}-c_{2}, 2 a_{3}-b_{3}-1\right) .
$$

В этом случае $b_{2}=c_{2}=1, a_{3}=\left(b_{3}+1\right) / 2$ и получается матрица, эквивалентная матрице вида (5.2).

Будем теперь предполагать, что $S$ - невырожденная система. Для получения необходимых и достаточных условий на коэффициенты матрицы $M\left(\gamma_{a}, \gamma_{b}, \gamma_{c}\right)$ будем применять теорему 4.

Пусть $S=\left(\gamma_{a}, \gamma_{b}, \gamma_{c}\right)$ - минимальная система с матрицей (5.4). Рассматривая линейные комбинации векторов $\gamma_{a}, \gamma_{b}, \gamma_{c}$ с коэффициентами (4.2), получаем, что дополнительные ограничения на коэффициенты матрицы $M\left(\gamma_{a}, \gamma_{b}, \gamma_{c}\right)$ возникают только из-за векторов

$$
\begin{aligned}
\gamma_{b}-\gamma_{c} & =\left(0, b_{2}+c_{2}, b_{3}+c_{3}\right), \\
\gamma_{a}-\gamma_{b}+\gamma_{c} & =\left(0,1-b_{2}-c_{2}, 1-b_{3}-c_{3}\right), \\
2 \gamma_{a}-\gamma_{b}+\gamma_{c} & =\left(0,2-b_{2}-c_{2}, 2-b_{3}-c_{3}\right) .
\end{aligned}
$$

Условия, что эти векторы не нарушают минимальности системы $S$, записываются соответственно в виде

$$
\begin{array}{lll}
b_{2}+c_{2} \geqslant 1 & \text { или } & b_{3}+c_{3} \geqslant 1, \\
b_{2}=c_{2}=1 & \text { или } & b_{3}=c_{3}=1, \\
b_{2}+c_{2} \leqslant 1 & \text { или } & b_{3}+c_{3} \leqslant 1 .
\end{array}
$$

В силу симметричности ситуации можно предположить, что $b_{3}=c_{3}=1, b_{2}+c_{2} \leqslant 1$. При этом матрица минимальной системы

$$
\left(\begin{array}{ccc}
0 & 1 & 1 \\
1 & b_{2} & -c_{2} \\
1 & 1 & -1
\end{array}\right), \quad \begin{gathered}
b_{2}, c_{2} \in(0,1], \\
b_{2}+c_{2} \leqslant 1
\end{gathered}
$$

является частным случаем матрицы (5.3).

Для матрицы (5.5) вектор $\gamma_{a}+\gamma_{c}=\left(c_{1}, 1-c_{2}, a_{3}-c_{3}\right)$ не нарушает минимальности системы $S$, только если $c_{1}=1$. При $c_{1}=1$ другие ограничения могут возникнуть только из-за векторов

$$
\gamma_{a}-\gamma_{b}+\gamma_{c}=\left(0,1-b_{2}-c_{2}, a_{3}-1-c_{3}\right), \quad 2 \gamma_{a}-\gamma_{b}+\gamma_{c}=\left(0,2-b_{2}-c_{2}, 2 a_{3}-1-c_{3}\right) .
$$

Условия, что эти векторы не нарушают минимальности $S$, записываются соответственно в виде

$$
\begin{array}{lll}
b_{2}=c_{2}=1 & \text { или } & a_{3} \leqslant c_{3}, \\
b_{2}+c_{2} \leqslant 1 & \text { или } & 2 a_{3} \leqslant c_{3} .
\end{array}
$$

При таких ограничениях мы приводим к матрицам вида (5.3). 
Для матрицы (5.6) вектор $\gamma_{a}-\gamma_{c}=\left(c_{1}, 1-c_{2}, a_{3}-1\right)$ не нарушает минимальности системы $S$ только при $c_{1}=1$. Но при $c_{1}=1$ вторая и третья матрицы эквивалентны, поэтому также получится матрица, эквивалентная (5.3).

Проверим достаточность условий теоремы. Для вырожденных систем она вытекает из теоремы 5. Для матриц вида (5.3) всегда выполняется неравенство $\operatorname{det} M_{6} \geqslant 1$. Действительно, при $a_{3} \leqslant c_{3} / 2$

$$
\operatorname{det} M_{6} \geqslant 1+c_{3}\left(1-\frac{b_{2}+c_{2}}{2}\right) \geqslant 1
$$

а при $a_{3} \leqslant c_{3}$ и $b_{2}+c_{2} \leqslant 1$

$$
\operatorname{det} M_{6} \geqslant 1+c_{3}\left(1-b_{2}-c_{2}\right) \geqslant 1 .
$$

Значит, система, задаваемая матрицей (5.3), является невырожденной, и по теореме 4 проверенные необходимые условия минимальности являются также и достаточными.

5.2. Минимальные системы без диагонального преобладания. В теореме 6 получена классификация минимальных систем, лежащих в двух октантах. Поэтому в дальнейшем будем рассматривать только такие тройки, векторы которых лежат в трех попарно различных октантах.

Теорема 7. Пусть $S=\left(\gamma_{a}, \gamma_{b}, \gamma_{c}\right)$ - система векторов решетки $\Gamma$, причем $\gamma_{a}, \gamma_{b}, \gamma_{c}$ лежат в трех различных октантах. Если система $S$ является минимальной системой без диагонального преобладания, то матрица $M\left(\gamma_{a}, \gamma_{b}, \gamma_{c}\right)$ с помощъю элементарных преобразований приводится к одной из следующих двух форм (см. рис. 3):

$$
\begin{aligned}
& M_{7}=\left(\begin{array}{rrr}
1 & 1 & c_{1} \\
a_{2} & -b_{2} & 1 \\
-a_{3} & -b_{3} & 1
\end{array}\right), \quad \begin{array}{c}
c_{1}=1, \quad 2 a_{2}+b_{2} \geqslant 2, \\
c_{1}=1, \quad a_{2}+b_{2} \geqslant 1, \quad b_{3} \leqslant 2 a_{3}, \\
\text { или } a_{2}+b_{2} \geqslant 1, \quad b_{3} \leqslant a_{3},
\end{array} \\
& M_{8}=\left(\begin{array}{rrr}
1 & 1 & c_{1} \\
a_{2} & -b_{2} & 1 \\
-a_{3} & b_{3} & 1
\end{array}\right), \quad a_{2}+b_{2} \geqslant 1,
\end{aligned}
$$

$u a_{2}, a_{3}, b_{2}, b_{3} \in[0,1), c_{1} \in[0,1]$.

$B$ первом случае система $S$ является базисом решетки Г. Во втором случае система $S$ может быть либо базисом, либо, при дополнительных условиях

$$
c_{1}=0, \quad a_{3}+b_{3} \geqslant 1,
$$

системой индекса 2 решетки $\Gamma=\left\langle\gamma_{a}, \gamma_{b},\left(\gamma_{a}+\gamma_{b}+\gamma_{c}\right) / 2\right\rangle$.

Наоборот, матрицы (5.7), (5.8) не эквивалентны матрицам с диагональным преобладанием; система $S=\left(\gamma_{a}, \gamma_{b}, \gamma_{c}\right)$ с матрицей (5.7) является минимальной системой решетки $\Gamma=\left\langle\gamma_{a}, \gamma_{b}, \gamma_{c}\right\rangle ;$ система $S=\left(\gamma_{a}, \gamma_{b}, \gamma_{c}\right)$ с матрицей (5.8) является минимальной системой решетки $\Gamma=\left\langle\gamma_{a}, \gamma_{b}, \gamma_{c}\right\rangle$, а при дополнительных условиях (5.9) и минимальной системой индекса 2 решетки $\Gamma=\left\langle\gamma_{a}, \gamma_{b},\left(\gamma_{a}+\gamma_{b}+\gamma_{c}\right) / 2\right\rangle$.

ДокаЗАТЕЛьство. Докажем необходимость условий теоремы. Матрицу системы $S=$ $\left(\gamma_{a}, \gamma_{b}, \gamma_{c}\right)$ нельзя привести к матрице с диагональным преобладанием элементов тогда и только тогда, когда два узла системы $S$ (например, $\gamma_{a}$ и $\gamma_{b}$ ) лежат внутри одной грани параллелепипеда $\Pi\left(\gamma_{a}, \gamma_{b}, \gamma_{c}\right)$, а третий узел (соответственно, $\left.\gamma_{c}\right)$ лежит на ребре, перпендикулярном этой грани. Поэтому достаточно рассматривать только минимальные системы $S=\left(\gamma_{a}, \gamma_{b}, \gamma_{c}\right)$ с матрицами вида

$$
\left(\begin{array}{ccc}
1 & 1 & c_{1} \\
\alpha_{2} a_{2} & \beta_{2} b_{2} & 1 \\
\alpha_{3} a_{3} & \beta_{3} b_{3} & 1
\end{array}\right), \quad \begin{gathered}
0 \leqslant c_{1} \leqslant 1 \\
\alpha_{2}, \alpha_{3}, \beta_{2}, \beta_{3}= \pm 1
\end{gathered}
$$




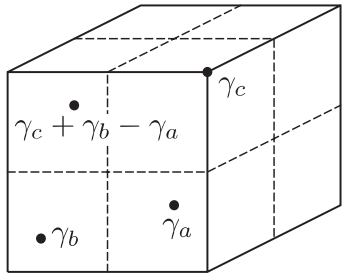

$M_{7}, \quad c_{1}=1$

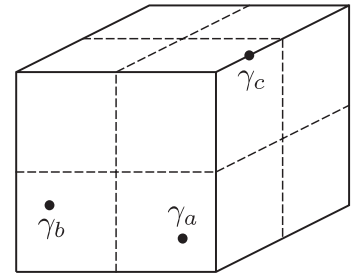

$M_{7}, \quad c_{1}<1$

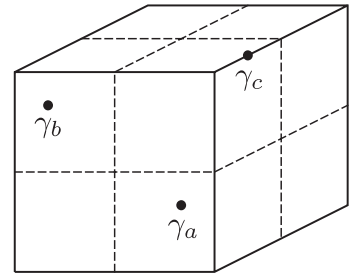

$M_{8}$

Рис. 3. Расположение точек минимальных систем с матрицами $M_{7}, M_{8}$

Заметим сначала, что $\left(\alpha_{2}, \alpha_{3}\right) \neq\left(\beta_{2}, \beta_{3}\right)$, так как в противном случае вектор $\gamma_{a}-\gamma_{b}=$ $\left(0, \alpha_{2}\left(a_{2}-b_{2}\right), \alpha_{3}\left(a_{3}-b_{3}\right)\right)$ нарушал бы минимальность системы $S$. Учитывая, что первые два столбца и последние две строки могут быть переставлены местами, получаем 4 возможных варианта выбора знаков:

$$
\left(\begin{array}{ll}
\alpha_{2} & \beta_{2} \\
\alpha_{3} & \beta_{3}
\end{array}\right)=\left(\begin{array}{ll}
1 & -1 \\
1 & -1
\end{array}\right), \quad\left(\begin{array}{rr}
1 & 1 \\
1 & -1
\end{array}\right), \quad\left(\begin{array}{rr}
1 & -1 \\
-1 & -1
\end{array}\right), \quad\left(\begin{array}{rr}
1 & -1 \\
-1 & 1
\end{array}\right) .
$$

В первом случае $a_{2} a_{3} b_{2} b_{3}=0$, так как иначе получается противоречие с предположением, что $\gamma_{a}, \gamma_{b}, \gamma_{c}$ лежат в трех различных октантах. После замены знака у нулевого элемента, получаем, что первый случай сводится ко второму или третьему.

Во втором случае вектор

$$
\gamma_{a}-\gamma_{c}=\left(1-c_{1}, a_{2}-1, a_{3}-1\right)
$$

не нарушает минимальность системы $S$, только если $c_{1} a_{2} a_{3}=0$. Если $c_{1}=0$, то второй случай с помощью замен знаков в строках и столбцах матрицы $M\left(\gamma_{a}, \gamma_{b}, \gamma_{c}\right)$ сводится к третьему, а если $a_{2}=0$, то к четвертому. Равенство $a_{3}=0$ невозможно, поскольку при $a_{3}=0$ вектор $\gamma_{a}-\gamma_{b}=\left(0, a_{2}-b_{2}, b_{3}\right)$ будет нарушать минимальность системы $S$.

В двух оставшихся вариантах проверку на вырожденность будем осуществлять с помощью теоремы 5. Для невырожденных систем необходимые условия на координаты векторов будем получать с помощью теоремы 4. Если тройка $\left(\gamma_{a}, \gamma_{b}, \gamma_{c}\right)$ окажется базисом, то найденные необходимые условия на коэффициенты будут также достаточными.

Рассмотрим случай, когда

$$
\left(\begin{array}{ll}
\alpha_{2} & \beta_{2} \\
\alpha_{3} & \beta_{3}
\end{array}\right)=\left(\begin{array}{rr}
1 & -1 \\
-1 & -1
\end{array}\right)
$$

Так как линейные комбинации векторов $\gamma_{a}, \gamma_{b}, \gamma_{c}$ с коэффициентами (4.5) не обращаются в нуль, то по теореме 5 система $S$ является невырожденной. Минимальность системы $S$ могут нарушать векторы

$$
\begin{aligned}
\gamma_{a}-\gamma_{b} & =\left(0, a_{2}+b_{2},-a_{3}+b_{3}\right) \\
\gamma_{b}-\gamma_{a}+\gamma_{c} & =\left(c_{1},-a_{2}-b_{2}+1, a_{3}-b_{3}+1\right), \\
2 \gamma_{a}-\gamma_{b}-\gamma_{c} & =\left(1-c_{1}, 2 a_{2}+b_{2}-1,-2 a_{3}+b_{3}-1\right) .
\end{aligned}
$$

Условия, что эти векторы не лежат внутри куба $\Pi=(-1,1)^{3}$, записываются соответственно в виде

$$
a_{2}+b_{2} \geqslant 1
$$

$$
\left.c_{1}=1 \quad \text { или } b_{3} \leqslant a_{3} \quad \text { (или } a_{2}=b_{2}=0\right),
$$

$$
c_{1}=0 \text { или } b_{3} \leqslant 2 a_{3}, \quad \text { или } 2 a_{2}+b_{2} \geqslant 2 \quad \text { (или } a_{2}=b_{2}=0 \text { ) }
$$


(в скобки взяты условия, противоречащие неравенству $a_{2}+b_{2} \geqslant 1$ ). Таким образом, матрица $M\left(\gamma_{a}, \gamma_{b}, \gamma_{c}\right)$ должна иметь вид (5.7).

Рассмотрим оставшийся случай

$$
\left(\begin{array}{ll}
\alpha_{2} & \beta_{2} \\
\alpha_{3} & \beta_{3}
\end{array}\right)=\left(\begin{array}{rr}
1 & -1 \\
-1 & 1
\end{array}\right) .
$$

Здесь также ни одна из линейных комбинаций векторов $\gamma_{a}, \gamma_{b}, \gamma_{c}$ с коэффициентами (4.5) не обращаются в нуль, и по теореме 5 система $S$ является невырожденной. Из линейных комбинаций векторов $\gamma_{a}, \gamma_{b}, \gamma_{c}$ с коэффициентами (4.2) минимальность системы $S$ может нарушать только вектор $\gamma_{a}-\gamma_{b}=\left(0, a_{2}+b_{2}, a_{3}+b_{3}\right)$. Условие $\gamma_{a}-\gamma_{b} \notin \Pi$ равносильно выполнению одного из двух эквивалентных неравенств $a_{2}+b_{2} \geqslant 1, a_{3}+b_{3} \geqslant 1$. Без ограничения общности можно считать, что $a_{2}+b_{2} \geqslant 1$. При таком ограничении матрица $M\left(\gamma_{a}, \gamma_{b}, \gamma_{c}\right)$ принимает вид (5.8).

Если $\left(\gamma_{a}, \gamma_{b}, \gamma_{c}\right)$ - базис решетки, то по теореме 4 необходимые условия минимальности описаны полностью.

Если же набор $\left(\gamma_{a}, \gamma_{b}, \gamma_{c}\right)$ не является базисом решетки, то по теореме 2 он образует систему индекса 2. При этом линейные комбинации векторов $\gamma_{a}, \gamma_{b}, \gamma_{c}$ с коэффициентами (4.3)

$$
\gamma_{1}=\frac{\gamma_{a}+\gamma_{b}+\gamma_{c}}{2}, \quad \gamma_{2}=\frac{-\gamma_{a}+\gamma_{b}+\gamma_{c}}{2}, \quad \gamma_{3}=\frac{\gamma_{a}-\gamma_{b}+\gamma_{c}}{2}, \quad \gamma_{4}=\frac{\gamma_{a}+\gamma_{b}-\gamma_{c}}{2}
$$

должны лежать вне П. Для матрицы (5.7) это невозможно, так как узел

$$
\gamma_{2}=\frac{1}{2}\left(c_{1}, 1-a_{2}-b_{2}, 1+a_{3}-b_{3}\right)
$$

нарушает минимальность $S$. Пусть столбцы матрицы (5.8) образуют систему индекса 2. Векторы

$$
\gamma_{1}=\frac{1}{2}\left(2-c_{1}, a_{2}-b_{2}-1,-a_{3}+b_{3}-1\right), \quad \gamma_{2}=\frac{1}{2}\left(c_{1}, 1-a_{2}-b_{2}, 1+a_{3}+b_{3}\right)
$$

не лежат в П тогда и только тогда, когда выполняются условия (5.9). При таких дополнительных ограничениях система $S=\left(\gamma_{a}, \gamma_{b}, \gamma_{c}\right)$ может быть как базисом решетки $\Gamma=\left\langle\gamma_{a}, \gamma_{b}, \gamma_{c}\right\rangle$, так и системой индекса 2 решетки $\Gamma=\left\langle\gamma_{a}, \gamma_{b},\left(\gamma_{a}+\gamma_{b}+\gamma_{c}\right) / 2\right\rangle$.

Докажем утверждение теоремы в другую сторону. Очевидно, что ни одна из систем с матрицами (5.7) или (5.8) не является системой с диагональным преобладанием. Проверим, что системы с матрицами (5.7) или (5.8) является невырожденными.

Для матриц вида (5.7)

$$
\left|\operatorname{det} M_{7}\right|=b_{2}-b_{3}+a_{2}+a_{3}+c_{1}\left(a_{2} b_{3}+a_{3} b_{2}\right) .
$$

Из условий $c_{1}=1, b_{2} \geqslant 2-2 a_{2}, b_{3}<1, a_{2}<1$ вытекает оценка

$$
\left|\operatorname{det} M_{7}\right| \geqslant 2-a_{2}-b_{3}+3 a_{3}+a_{2} b_{3}-2 a_{2} a_{3} \geqslant 1+\frac{a_{2} b_{3}}{2} \geqslant 1 \text {. }
$$

Если $c_{1}=1, b_{2} \geqslant 1-a_{2}, a_{3} \geqslant b_{3} / 2$, то

$$
\left|\operatorname{det} M_{7}\right| \geqslant 1-b_{3}+2 a_{3}+a_{2} b_{3}-a_{2} a_{3} \geqslant 1+\frac{a_{2} b_{3}}{2} \geqslant 1 .
$$

При $c_{1}=0, a_{2}+b_{2} \geqslant 1, a_{3} \geqslant b_{3}$

$$
\left|\operatorname{det} M_{7}\right| \geqslant a_{2}+b_{2}+a_{3}-b_{3} \geqslant 1 .
$$


Для матриц вида (5.8) из условий $a_{2}+b_{2} \geqslant 1, c_{1} a_{2} \leqslant 1$ получаем оценку

$$
\left|\operatorname{det} M_{8}\right|=b_{2}+b_{3}+a_{2}+a_{3}+c_{1}\left(a_{3} b_{2}-a_{2} b_{3}\right) \geqslant 1 \text {. }
$$

Таким образом, системы векторов с матрицами (5.7), (5.8) являются невырожденными. Кроме того, для рассматриваемых решеток $\Gamma=\left\langle\gamma_{a}, \gamma_{b}, \gamma_{c}\right\rangle$ и $\Gamma=\left\langle\gamma_{a}, \gamma_{b},\left(\gamma_{a}+\gamma_{b}+\gamma_{c}\right) / 2\right\rangle$ октаэдр с вершинами $\pm \gamma_{a}, \pm \gamma_{b}, \pm \gamma_{c}$ является примитивным. Значит, к тройке векторов $\left(\gamma_{a}, \gamma_{b}, \gamma_{c}\right)$ применима теорема 4, и найденные необходимые условия на коэффициенты матриц становятся и достаточными условиями минимальности.

5.3. Минимальные системы стандартного вида. В теоремах 6,7 описаны минимальные системы, которые содержатся в двух октантах или не записываются в виде матрицы с диагональным преобладанием. Для завершения классификации минимальных систем остается рассмотреть случай систем стандартного вида (2.3). Как отмечалось выше, матрицы таких систем приводятся к одной из канонических форм (напомним, что нулевым координатам знак может быть присвоен произвольно)

$$
\begin{aligned}
& \left(\begin{array}{rrr}
1 & b_{1} & -c_{1} \\
-a_{2} & 1 & c_{2} \\
a_{3} & -b_{3} & 1
\end{array}\right), \\
& \left(\begin{array}{ccc}
1 & b_{1} & c_{1} \\
-a_{2} & 1 & c_{2} \\
a_{3} & -b_{3} & 1
\end{array}\right), \\
& \left(\begin{array}{ccc}
1 & -b_{1} & -c_{1} \\
-a_{2} & 1 & -c_{2} \\
-a_{3} & -b_{3} & 1
\end{array}\right)
\end{aligned}
$$

где $a_{i}, b_{i}, c_{i} \in[0,1]$.

Рассмотрим отдельно исключительные минимальные системы стандартного вида с индексами 2, 0 и 1 (другие варианты невозможны по теореме 2).

\subsection{1. Исключительные минимальные системы стандартного вида индекса 2.}

Теорема 8. Пусть $S=\left(\gamma_{a}, \gamma_{b}, \gamma_{c}\right)$ - минимальная система стандартного вида (2.3), имеющая в решетке Г индекс 2. Тогда матрища $M\left(\gamma_{a}, \gamma_{b}, \gamma_{c}\right)$ эквивалентна одной из двух матрии, (см. рис. 4)

$$
\begin{aligned}
M_{9}=\left(\begin{array}{rrr}
1 & 1 & 0 \\
-1 & 1 & 0 \\
a_{3} & -b_{3} & 1
\end{array}\right), & a_{3}, b_{3} \in[0,1], \\
M_{10}=\left(\begin{array}{rrr}
1 & 1 & 0 \\
-a_{2} & 1 & c_{2} \\
a_{3} & -b_{3} & 1
\end{array}\right), & a_{2}, c_{2}, a_{3}, b_{3} \in[0,1],
\end{aligned}
$$

$u \Gamma=\left\langle\gamma_{a}, \gamma_{b},\left(\gamma_{a}+\gamma_{b}+\gamma_{c}\right) / 2\right\rangle$.

Наоборот, каждая из матрии (5.14), (5.15) задает минимальную систему индекса 2 в решетке $\Gamma=\left\langle\gamma_{a}, \gamma_{b},\left(\gamma_{a}+\gamma_{b}+\gamma_{c}\right) / 2\right\rangle$.

ДокАЗАТЕЛЬСтво. По теореме 4 линейно независимая система $S=\left(\gamma_{a}, \gamma_{b}, \gamma_{c}\right)$ будет минимальной системой индекса 2 в решетке $\Gamma=\left\langle\gamma_{a}, \gamma_{b},\left(\gamma_{a}+\gamma_{b}+\gamma_{c}\right) / 2\right\rangle$ тогда и только тогда, когда векторы (5.10) не лежат в кубе $\Pi=(-1,1)^{3}$.

Для матриц вида (5.13)

$$
\gamma_{1}=\left(\frac{1-b_{1}-c_{1}}{2}, \frac{1-a_{2}-c_{2}}{2}, \frac{1-a_{3}-b_{3}}{2}\right) \in \Pi,
$$

поэтому в условиях теоремы такая расстановка знаков невозможна. 


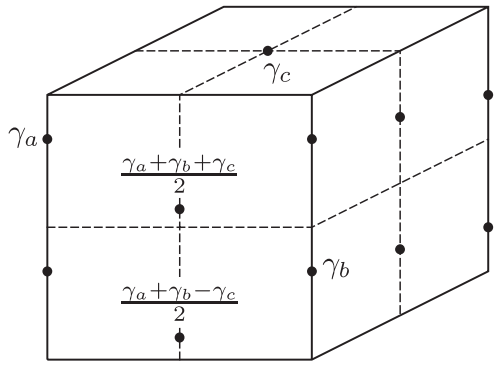

$M_{9}$

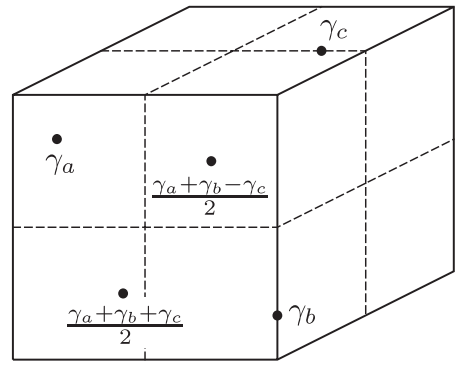

$M_{10}$

Рис. 4. Расположение точек минимальных систем с матрицами $M_{9}, M_{10}$

Для матриц вида (5.11) вектор

$$
\gamma_{1}=\left(\frac{1+b_{1}-c_{1}}{2}, \frac{1-a_{2}+c_{2}}{2}, \frac{1+a_{3}-b_{3}}{2}\right)
$$

не лежит в П, если выполнено по крайней мере одно из трех неравенств

$$
b_{1}-c_{1} \geqslant 1, \quad c_{2}-a_{2} \geqslant 1, \quad a_{3}-b_{3} \geqslant 1 .
$$

Действием группы $G_{3}$ эти неравенства могут быть переведены друг в друга. Поэтому без ограничения общности можно считать, что выполнено первое неравенство, т.е. $b_{1}=1, c_{1}=0$.

Для матриц вида (5.12) вектор

$$
\gamma_{4}=\left(\frac{1+b_{1}-c_{1}}{2}, \frac{1-a_{2}-c_{2}}{2}, \frac{-1+a_{3}-b_{3}}{2}\right)
$$

не лежит в П, если выполнено по крайней мере одно из двух неравенств

$$
b_{1}-c_{1} \geqslant 1, \quad a_{3}-b_{3} \leqslant-1 .
$$

Эти неравенства также переводятся друг в друга действием группы $G_{3}$. Значит, снова можно предположить справедливость первого неравенства, из которого следует, что $b_{1}=1, c_{1}=0$. Следовательно, во всех случаях матрица $M\left(\gamma_{a}, \gamma_{b}, \gamma_{c}\right)$ может быть приведена к виду

$$
\left(\begin{array}{ccc}
1 & 1 & 0 \\
-a_{2} & 1 & c_{2} \\
a_{3} & -b_{3} & 1
\end{array}\right)
$$

Для такой матрицы $\gamma_{1}, \gamma_{4} \notin \Pi$, и остается рассмотреть векторы $\gamma_{2}$ и $\gamma_{3}$. Условие

$$
\gamma_{3}=\left(0, \frac{-1-a_{2}+c_{2}}{2}, \frac{1+a_{3}+b_{3}}{2}\right) \notin \Pi
$$

равносильно выполнению одного из неравенств

$$
c_{2}+1 \leqslant a_{2}, \quad a_{3}+b_{3} \geqslant 1 .
$$

Если выполнено первое неравенство, то $a_{2}=1, c_{2}=0$. При этом

$$
\gamma_{2}=\left(0,1, \frac{1-a_{3}-b_{3}}{2}\right) \notin \Pi,
$$


и других ограничений на координаты векторов не возникает. Таким образом, получается множество матриц вида (5.14). Если же выполнено неравенство $a_{3}+b_{3} \geqslant 1$, то условие

$$
\gamma_{2}=\left(0, \frac{a_{2}+c_{2}+1}{2}, \frac{1-a_{3}-b_{3}}{2}\right) \notin \Pi
$$

равносильно неравенству $a_{2}+c_{2} \geqslant 1$. В этом случае матрица $M\left(\gamma_{a}, \gamma_{b}, \gamma_{c}\right)$ принадлежит множеству матриц (5.15).

Докажем утверждение теоремы в обратную сторону. Поскольку $\operatorname{det} M_{9}=2$ и

$$
\operatorname{det} M_{10}=1+a_{2}+c_{2}\left(a_{3}+b_{3}\right) \geqslant 1+a_{2}+c_{2} \geqslant 2
$$

то каждая из матриц (5.14), (5.15) не вырождена и имеет индекс 2 в решетке $\Gamma=\left\langle\gamma_{a}, \gamma_{b}\right.$, $\left.\left(\gamma_{a}+\gamma_{b}+\gamma_{c}\right) / 2\right\rangle$. По теореме 4 проверенные необходимые условия минимальности являются также и достаточными.

\subsection{2. Вырожденные исключительные минимальные системы стандартного вида.}

ТеОрема 9. Пусть $S=\left(\gamma_{a}, \gamma_{b}, \gamma_{c}\right)$ - вырожденная минимальная система стандартного вида решетки Г. Тогда матрица $M\left(\gamma_{a}, \gamma_{b}, \gamma_{c}\right)$ эквивалентна матрице (см. рис. 5)

$$
M_{11}=\left(\begin{array}{crr}
1 & -1 & -1 \\
0 & 1 & -1 \\
\frac{b_{3}-1}{2} & -b_{3} & 1
\end{array}\right), \quad b_{3} \in[0,1] .
$$

Наоборот, система векторов, матрица которой приводится $\kappa$ виду (5.16), является върожденной минимальной системой стандартного вида решетки Г.

Верно и обратное: система из трех векторов $\left(\gamma_{a}, \gamma_{b}, \gamma_{c}\right)$, матрища которой приводится $\kappa$ виду (5.16), является минимальной системой решетки (ранга 2) $\Gamma=\left\langle\gamma_{a}, \gamma_{b}, \gamma_{c}\right\rangle=\left\langle\gamma_{b}, \gamma_{c}\right\rangle$.

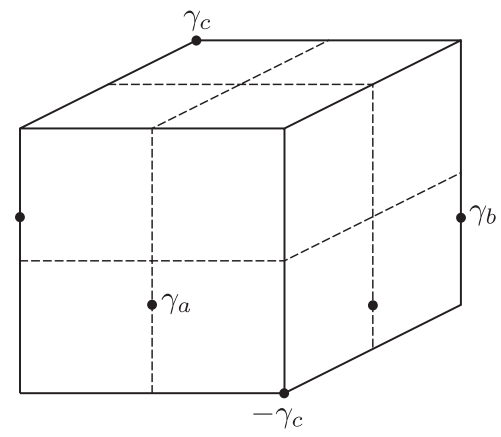

Рис. 5. Расположение точек минимальной системы с матрицей $M_{11}$

ДокАЗАТЕЛЬство. Если матрица системы $S$ имеет вид (5.11), то среди линейных комбинаций векторов $\gamma_{a}, \gamma_{b}, \gamma_{c}$ с коэффициентами (4.5) нулевым может быть только вектор $\gamma_{a}+\gamma_{b}+\gamma_{c}=\left(1+b_{1}-c_{1}, 1-a_{2}+c_{2}, 1+a_{3}-b_{3}\right)$. Это возможно только при $a_{3}=b_{1}=c_{2}=0$, $a_{2}=b_{3}=c_{1}=1$. Но в таком случае данная минимальная система не будет исключительной, поскольку она является частным случаем минимальной системы (2.6).

Если матрица $M\left(\gamma_{a}, \gamma_{b}, \gamma_{c}\right)$ имеет вид (5.12), то среди линейных комбинаций векторов $\gamma_{a}$, $\gamma_{b}, \gamma_{c}$ с коэффициентами (4.5) в нуль могут обращаться только векторы

$$
\begin{aligned}
\gamma_{a}+\gamma_{b}-\gamma_{c} & =\left(1+b_{1}-c_{1}, 1-a_{2}-c_{2}, a_{3}-b_{3}-1\right), \\
\gamma_{a}+2 \gamma_{b}-\gamma_{c} & =\left(1+2 b_{1}-c_{1}, 2-a_{2}-c_{2}, a_{3}-2 b_{3}-1\right) .
\end{aligned}
$$


Если $\gamma_{a}+\gamma_{b}-\gamma_{c}=0$, то $b_{1}=b_{3}=0, c_{1}=a_{3}=0, a_{2}+c_{2}=1$ и матрица

$$
M\left(\gamma_{a}, \gamma_{b}, \gamma_{c}\right)=\left(\begin{array}{ccc}
1 & 0 & 1 \\
-a_{2} & 1 & 1-a_{2} \\
1 & 0 & 1
\end{array}\right)
$$

эквивалентна матрице (2.6). Таким образом, система $S$ также не является исключительной. Если же $\gamma_{a}+2 \gamma_{b}+\gamma_{c}=0$, то $b_{1}=b_{3}=0, c_{1}=a_{3}=0, a_{2}=c_{2}=1$ и матрица

$$
M\left(\gamma_{a}, \gamma_{b}, \gamma_{c}\right)=\left(\begin{array}{rrr}
1 & 0 & 1 \\
-1 & 1 & 1 \\
1 & 0 & 1
\end{array}\right)
$$

эквивалентна матрице $(5.16)$, в которой $b_{3}=1$.

Предположим теперь, что исключительной является система с матрицей (5.13). Тогда по определению исключительной системы равенство $\gamma_{a}+\gamma_{b}+\gamma_{c}=0$ выполняться не может. Среди линейных комбинаций векторов $\gamma_{a}, \gamma_{b}, \gamma_{c}$ с коэффициентами (4.5) нулевым может быть только один из векторов

$$
2 \gamma_{a}+\gamma_{b}+\gamma_{c}, \quad \gamma_{a}+2 \gamma_{b}+\gamma_{c}, \quad \gamma_{a}+\gamma_{b}+2 \gamma_{c} .
$$

Из симметричности ситуации следует, что нулевым можно считать первый вектор. Тогда $b_{1}=c_{1}=1,2 a_{2}+c_{2}=1,2 a_{3}+c_{3}=1$. Из линейных комбинаций векторов $\gamma_{a}, \gamma_{b}, \gamma_{c}$ с коэффициентами (4.4) дополнительные ограничения на координаты возникают из-за векторов

$$
\gamma_{a}+\gamma_{b}=\left(0,1-a_{2},-a_{3}-b_{3}\right), \quad \gamma_{a}+\gamma_{c}=\left(0, c_{2}-a_{2}, 1-a_{3}\right) .
$$

Требования $\gamma_{a}+\gamma_{b}, \gamma_{a}+\gamma_{c} \notin$ П равносильны выполнению соответственно условий

$$
\begin{gathered}
a_{2}=0 \quad \text { или } a_{3}+b_{3} \geqslant 1, \\
\left(c_{2}=1, a_{2}=0\right) \text { или } \quad\left(c_{2}=0, a_{2}=1\right), \quad \text { или } a_{3}=0 .
\end{gathered}
$$

Поэтому возникает два варианта решений:

$$
\begin{array}{lll}
b_{1}=c_{1}=c_{2}=1, & a_{2}=0, & 2 a_{3}+b_{3}=1 ; \\
b_{1}=c_{1}=b_{3}=1, & a_{3}=0, & 2 a_{2}+b_{2}=1 .
\end{array}
$$

Обе соответствующие им матрицы

$$
\left(\begin{array}{crr}
1 & -1 & -1 \\
0 & 1 & -1 \\
\frac{b_{3}-1}{2} & -b_{3} & 1
\end{array}\right) ; \quad\left(\begin{array}{crr}
1 & -1 & -1 \\
\frac{c_{2}-1}{2} & 1 & -c_{2} \\
0 & -1 & 1
\end{array}\right)
$$

эквивалентны матрице (5.16).

Докажем утверждение теоремы в обратную сторону. Система векторов с матрицей (5.16) вырождена $\left(2 \gamma_{a}+\gamma_{b}+\gamma_{c}=0\right)$ и порождает решетку $\Gamma=\left\langle\gamma_{b}, \gamma_{c}\right\rangle$. По теореме 5 проверенные необходимые условия минимальности являются также и достаточными.

\subsection{3. Исключительные минимальные базисы стандартного вида.}

ТЕОРема 10. Пусть $S=\left(\gamma_{a}, \gamma_{b}, \gamma_{c}\right)$ - минимальная система стандартного вида решетки Г. Если $S$ является базисом решетки $\Gamma$, то матрица $M\left(\gamma_{a}, \gamma_{b}, \gamma_{c}\right)$ эквивалентна матрице (cм.puc. 6)

$$
M_{12}=\left(\begin{array}{rrr}
1 & -1 & -1 \\
-a_{2} & 1 & -c_{2} \\
-a_{3} & -b_{3} & 1
\end{array}\right), \quad \begin{gathered}
a_{2}, a_{3}, b_{3}, c_{2} \in[0,1], \\
2 a_{2}+b_{2} \geqslant 2, \quad a_{3}+b_{3} \geqslant 1
\end{gathered}
$$

Наоборот, всякая система с матрицей, эквивалентной (5.17), является минималъной системой решетки $\Gamma=\left\langle\gamma_{a}, \gamma_{b}, \gamma_{c}\right\rangle$. 


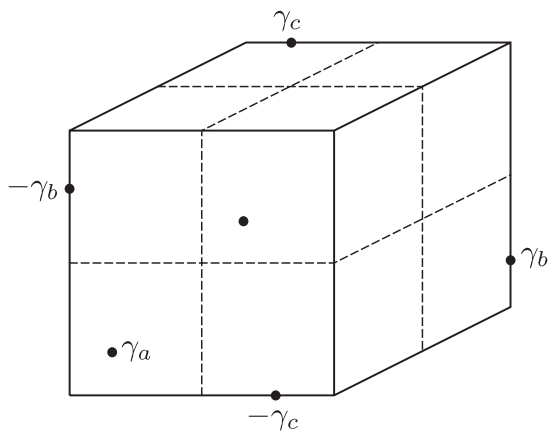

Рис. 6. Расположение точек минимальной системы с матрицей $M_{12}$

ДоказАтеЛьство. Как отмечалось выше, матрица $M\left(\gamma_{a}, \gamma_{b}, \gamma_{c}\right)$ с помощью элементарных преобразований может быть приведена к одной из канонических форм (5.11)-(5.13). По определению базисов Минковского минимальная система, являющаяся базисом, может быть исключительной, только если ее матрица эквивалентна матрице (5.13).

Как и раньше, для нахождения необходимых и достаточных условий на коэффициенты матрицы $M(S)$ будем рассматривать линейные комбинации векторов $\gamma_{a}, \gamma_{b}, \gamma_{c}$ с коэффициентами (4.2). Условие

$$
\gamma_{a}+\gamma_{b}+\gamma_{c}=\left(1-b_{1}-c_{1}, 1-a_{2}-c_{2}, 1-a_{3}-b_{3}\right) \notin \Pi
$$

равносильно выполнению одного из неравенств

$$
\left|1-b_{1}-c_{1}\right| \geqslant 1, \quad\left|1-a_{2}-c_{2}\right| \geqslant 1, \quad\left|1-a_{3}-b_{3}\right| \geqslant 1 .
$$

Так как эти неравенства эквивалентны, будем предполагать, что выполняется первое из них. Это возможно, если $b_{1}=c_{1}=0$ или $b_{1}=c_{1}=1$. Первый случай невозможен: при $b_{1}=$ $c_{1}=0$ система $S$ не является исключительной, так как присваивая коэффициентам $b_{1}$ и $c_{1}$ положительные знаки, мы получаем матрицу базиса Минковского II типа (сигнатура 13 из табл. 1). Поэтому далее будем предполагать, что $b_{1}=c_{1}=1$. Из линейных комбинаций векторов $\gamma_{a}, \gamma_{b}, \gamma_{c}$ с коэффициентами (4.2) минимальность системы $S$ могут нарушать только векторы $\gamma_{a}+\gamma_{b}, \gamma_{a}+\gamma_{c}, 2 \gamma_{a}+\gamma_{b}+\gamma_{c}$. Рассмотрим вектор

$$
2 \gamma_{a}+\gamma_{b}+\gamma_{c}=\left(0,1-2 a_{2}-2 c_{2}, 1-2 a_{3}-2 c_{3}\right) .
$$

Он не нарушает минимальности системы $S$ тогда и только тогда, когда выполняется по крайней мере одно из следующих четырех условий:

$$
a_{2}=c_{2}=0, \quad a_{3}=b_{3}=0, \quad 2 a_{2}+c_{2} \geqslant 2, \quad 2 a_{3}+b_{3} \geqslant 2 .
$$

Два первых условия и два последних эквивалентны друг другу. Поэтому достаточно рассмотреть два случая: $a_{2}=c_{2}=0$ и $2 a_{2}+c_{2} \geqslant 2$. Первый из них невозможен, так как при $a_{2}=c_{2}=0$ система $S$ будет базисом Минковского, а значит, не будет исключительной системой. Во втором случае $\gamma_{a}+\gamma_{c} \notin \Pi$, а условие $\gamma_{a}+\gamma_{b}=\left(0,1-a_{2},-a_{3}-b_{3}\right) \notin \Pi$ равносильно неравенству $a_{3}+b_{3} \geqslant 1$. Таким образом, матрица $M\left(\gamma_{a}, \gamma_{b}, \gamma_{c}\right)$ должна иметь вид (5.17).

Предположим, что система $S$ имеет матрицу (5.17). Тогда система $S$ невырожденная. Действительно,

$$
-\operatorname{det} M_{12}=-1+a_{2}+a_{3}+c_{2} b_{3}+c_{2} a_{3}+b_{3} a_{2} .
$$

Пользуясь неравенствами $c_{2} \geqslant 2-2 a_{2}, b_{3} \geqslant 1-a_{3}$, приходим к оценке

$$
-\operatorname{det} M_{12} \geqslant 1+a_{3}\left(1-a_{2}\right) \geqslant 1 \text {. }
$$


Значит, по теореме 4 проверенные необходимые условия минимальности являются также и достаточными.

\section{6. Трехмерный аналог теоремы Валена для приводимых решеток}

С помощью классификации минимальных систем, полученной в теоремах 6-10, трехмерный аналог теоремы Валена (1.3) может быть распространен на произвольные решетки следующим образом.

ТЕОРема 11. Если $S=\left(\gamma_{a}, \gamma_{b}, \gamma_{c}\right)$ - вполне минимальная система решетки Г и матрица $M\left(\gamma_{a}, \gamma_{b}, \gamma_{c}\right)$ имеет вид $(2.2)$, то

$$
a_{1} a_{2} a_{3}+b_{1} b_{2} b_{3}+c_{1} c_{2} c_{3} \leqslant \operatorname{det} \Gamma .
$$

Для произвольной минимальной системы $S=\left(\gamma_{a}, \gamma_{b}, \gamma_{c}\right)$ выполняется неравенство

$$
a_{1} a_{2} a_{3}+b_{1} b_{2} b_{3}+c_{1} c_{2} c_{3} \leqslant 2 \operatorname{det} \Gamma .
$$

ЗАмечаниЕ 2 . Если $S=\left(\gamma_{a}, \gamma_{b}, \gamma_{c}\right)$ - минимальная система решетки $Г$, то по теореме Минковского о выпуклом теле $\operatorname{vol}(\Pi(S)) \leqslant \operatorname{det} \Gamma$. Кроме того,

$$
\max \left\{a_{1} a_{2} a_{3}, b_{1} b_{2} b_{3}, c_{1} c_{2} c_{3}\right\} \leqslant \operatorname{vol}(\Pi(S)) .
$$

Поэтому всегда выполняется тривиальная оценка (1.4), и теорему 11 можно рассматривать как уточнение этой оценки.

ДоКАЗАТЕЛЬСТвО ТЕОРЕМЫ 11. Для матрицы $M$, записанной в виде $(2.2)$, определим функции

$$
\Delta_{k}(M)=k \operatorname{det} \Gamma-a_{1} a_{2} a_{3}-b_{1} b_{2} b_{3}-c_{1} c_{2} c_{3}, \quad k=1,2 .
$$

Для матриц стандартного вида $(2.3)$ функции $\Delta_{k}(M)$ будут иметь вид

$$
\Delta_{k}(M)=k \operatorname{det} \Gamma-a_{2} a_{3}-b_{1} b_{3}-c_{1} c_{2}, \quad k=1,2 .
$$

Докажем, что для минимальных троек выполняется неравенство $\Delta_{2}(M) \geqslant 0$, а для вполне минимальных - неравенство $\Delta_{1}(M) \geqslant 0$.

Для минимальных систем Минковского (2.4)-(2.6) неравенство $\Delta_{1}(M) \geqslant 0$ может быть доказано так же, как и в случае неприводимых решеток (см. [21]). Действительно, для матриц вида (2.4) из неравенств $b_{1} \leqslant 1, b_{1} \leqslant c_{1}$ следуют оценки

$$
\begin{aligned}
\Delta_{1}\left(M_{1}\right) & =1+c_{2} b_{3}-a_{2} a_{3}+b_{1}\left(a_{2}+c_{2} a_{3}-b_{3}\right)-c_{1}\left(c_{2}+a_{2} b_{3}-a_{3}\right) \\
& \geqslant b_{1}\left(1+c_{2} b_{3}-a_{2} a_{3}+a_{2}+c_{2} a_{3}-b_{3}\right)-c_{1}\left(c_{2}+a_{2} b_{3}-a_{3}\right) \\
& \geqslant c_{1}\left(1+c_{2} b_{3}-a_{2} a_{3}+a_{2}+c_{2} a_{3}-b_{3}-c_{2}-a_{2} b_{3}+a_{3}\right) \\
& =c_{1}\left(a_{3}\left(1-a_{2}\right)+a_{2}\left(1-b_{3}\right)+\left(1-c_{2}\right)\left(1-b_{3}\right)+c_{2} a_{3}\right) \geqslant 0 .
\end{aligned}
$$

Аналогично, для матриц вида $(2.5)$ из неравенств $b_{1} \leqslant 1, b_{1} \leqslant c_{1}, a_{2}+c_{2} \geqslant 1$ следует, что

$$
\begin{aligned}
\Delta_{1}\left(M_{2}\right) & =1+c_{2} b_{3}-a_{2} a_{3}+b_{1}\left(a_{2}+c_{2} a_{3}-b_{3}\right)+c_{1}\left(a_{2} b_{3}-a_{3}-c_{2}\right) \\
& \geqslant b_{1}\left(1+c_{2} b_{3}-a_{2} a_{3}+a_{2}+c_{2} a_{3}-b_{3}\right)+c_{1}\left(a_{2} b_{3}-a_{3}-c_{2}\right) \\
& \geqslant c_{1}\left(1+c_{2} b_{3}-a_{2} a_{3}+a_{2}+c_{2} a_{3}-b_{3}+a_{2} b_{3}-a_{3}-c_{2}\right) \\
& =c_{1}\left(\left(1-c_{2}\right)\left(1-a_{3}\right)+a_{2}\left(1-a_{3}\right)+b_{3}\left(a_{2}+c_{2}-1\right)\right) \geqslant 0 .
\end{aligned}
$$

Для минимальной системы с матрицей (2.6)

$$
a_{2} a_{3}+b_{1} b_{3}+c_{1} c_{2} \leqslant\left(b_{1}+c_{1}\right)\left(a_{2}+c_{2}\right)\left(a_{3}+b_{3}\right)=1 .
$$


По теореме Минковского о выпуклом теле (см. замечание 2$) \operatorname{det} \Gamma \geqslant 1$. Следовательно, $a_{2} a_{3}+$ $b_{1} b_{3}+c_{1} c_{2} \leqslant 1 \leqslant \operatorname{det} \Gamma$ и $\Delta_{1}\left(M_{3}\right) \geqslant 0$.

Перейдем к анализу исключительных минимальных систем. Рассмотрим сначала минимальные системы, лежащие в двух октантах. Снова применяя неравенство $\operatorname{det} \Gamma \geqslant 1$, для матрицы вида (5.1) получаем оценку

$$
\Delta_{1}\left(M_{4}\right) \geqslant 1-b_{2} b_{3}-\left(1-b_{2}\right)\left(1-b_{3}\right)=b_{2}+b_{3}-b_{2} b_{3} \geqslant 0 .
$$

Аналогично, для матрицы вида (5.2)

$$
\Delta_{2}\left(M_{5}\right)=2 \operatorname{det} \Gamma-1-c_{3} \geqslant 1-c_{3} \geqslant 0 .
$$

Система с матрицей (5.2) не может быть вполне минимальной, так как $\gamma_{a}$ нарушает минимальность $\gamma_{b}$.

Для матрицы вида (5.3)

$$
\Delta_{2}\left(M_{6}\right)=2+2 c_{3}-2 a_{3}\left(b_{2}+c_{2}\right)-b_{2}-c_{2} c_{3} .
$$

Если $a_{3} \leqslant c_{3} / 2$, то

$$
\Delta_{2}\left(M_{6}\right) \geqslant c_{3}\left(2-b_{2}-c_{2}\right)+2-b_{2}-c_{2} c_{3} \geqslant 0 .
$$

Если же $a_{3} \leqslant c_{3}$ и $c_{2} \leqslant 1-b_{2}$, то

$$
\Delta_{2}\left(M_{6}\right) \geqslant 2-b_{2}-c_{3}+b_{2} c_{3} \geqslant 1 \text {. }
$$

Пусть матрица (5.3) задает вполне минимальную систему. Тогда $c_{2} \neq 1$ (в противном случае $\gamma_{a}$ нарушает минимальность $\left.\gamma_{c}\right)$. Значит, вектор

$$
\gamma_{a}-\gamma_{b}+\gamma_{c}=\left(0,1-b_{2}-c_{2}, a_{3}-1-c_{3}\right)
$$

не будет нарушать минимальность узла $\gamma_{b}=\left(1, b_{2}, 1\right)$, только если $b_{2} \leqslant\left(1-c_{2}\right) / 2$. При таком ограничении, учитывая неравенство $a_{3} \leqslant c_{3}$, получаем оценку

$$
\Delta_{1}\left(M_{6}\right) \geqslant \frac{1}{2}\left(1+c_{2}+c_{3}-3 c_{2} c_{3}\right) \geqslant 0
$$

Рассмотрим теперь минимальные системы без диагонального преобладания. Если в матрице $(5.7) c_{1}=1$, то система $\left(\gamma_{a}, \gamma_{b}, \gamma_{c}\right)$ не является вполне минимальной $\left(\gamma_{a}\right.$ и $\gamma_{b}$ нарушают минимальность $\gamma_{c}$ ), и достаточно доказать справедливость неравенства (6.2).

Предположим, что $2 a_{2}+b_{2} \geqslant 2$. В представлении

$$
\Delta_{2}\left(M_{7}\right)=2\left(a_{2}+a_{3}+b_{2}-b_{3}+a_{2} b_{3}+a_{3} b_{2}\right)-a_{2} a_{3}-b_{2} b_{3}-1
$$

коэффициент при $b_{2}$ положителен, а при $b_{3}$ отрицателен. Поэтому оценку $\Delta_{2}\left(M_{7}\right) \geqslant 0$ достаточно проверить при $b_{2}=2-2 a_{2}$ и $b_{3}=1$. В этом случае

$$
\Delta_{2}\left(M_{7}\right)=2 a_{2}-1+6 a_{3}-5 a_{2} a_{3} \geqslant 2 a_{2}-1 \geqslant 0,
$$

так как неравенство $2 a_{2}+b_{2} \geqslant 2$ может выполняться только при $a_{2} \geqslant 1 / 2$.

Предположим, что $a_{2}+b_{2} \geqslant 1$ и $a_{3} \geqslant b_{3} / 2$. В равенстве (6.3) коэффициент при $a_{3}$ отрицателен, а при $b_{2}$, как это уже отмечалось выше, положителен. Значит, оценку $\Delta_{2}\left(M_{7}\right) \geqslant 0$ достаточно проверить при $b_{2}=1-a_{2}$ и $a_{3}=b_{3} / 2$. Для таких значений $b_{2}$ и $a_{3}$

$$
\Delta_{2}\left(M_{7}\right)=1-b_{3}+\frac{3}{2} a_{2} b_{3} \geqslant 1-b_{3} \geqslant 0
$$


Рассмотрим случай, когда элементы матрицы (5.7) связаны неравенствами $a_{2}+b_{2} \geqslant 1$, $b_{3} \leqslant a_{3}$. В разложении

$$
\Delta_{1}\left(M_{7}\right)=a_{2}+a_{3}+b_{2}-b_{3}+c_{1}\left(a_{2} b_{3}+a_{3} b_{2}-1\right)-a_{2} a_{3}-b_{2} b_{3}
$$

коэффициент при $b_{2}$ положителен, а при $b_{3}$ отрицателен. Поэтому оценку $\Delta_{1}\left(M_{7}\right) \geqslant 0$ достаточно проверить при $b_{2}=1-a_{2}$ и $b_{3}=a_{3}$. В этом случае

$$
\Delta_{1}\left(M_{7}\right)=\left(1-a_{3}\right)\left(1-c_{1}\right) \geqslant 0 .
$$

Если минимальная система с матрицей (5.8) является базисом решетки, то $\left|\operatorname{det} M_{8}\right|=$ $\operatorname{det} \Gamma$ и

$$
\Delta_{1}\left(M_{8}\right)=a_{2}+a_{3}+b_{2}+b_{3}+c_{1}\left(a_{3} b_{2}-a_{2} b_{3}\right)-a_{2} a_{3}-b_{2} b_{3}-c_{1} .
$$

Здесь коэффициент при $b_{2}$ положителен, а при $c_{1}$ отрицателен. Поэтому оценку $\Delta_{1}\left(M_{8}\right) \geqslant 0$ достаточно проверить при $c_{1}=1$ и $b_{2}=1-a_{2}$. В этом случае

$$
\Delta_{1}\left(M_{8}\right)=2 a_{3}\left(1-a_{2}\right) \geqslant 0 .
$$

Если же минимальная система имеет индекс 2 , то $\left|\operatorname{det} M_{8}\right|=2 \operatorname{det} \Gamma$, и при этом выполняется дополнительное условие $c_{1}=0$. Значит,

$$
\Delta_{1}\left(M_{8}\right)=\frac{1}{2}\left|\operatorname{det} M_{5}\right|-a_{2} a_{3}-b_{2} b_{3}=\frac{1}{2}\left(a_{2}+a_{3}+b_{2}+b_{3}\right)-a_{2} a_{3}-b_{2} b_{3} \geqslant 0,
$$

поскольку $b_{2} \geqslant b_{2} b_{3}, b_{3} \geqslant b_{2} b_{3}, a_{2} \geqslant a_{2} a_{3}, a_{3} \geqslant a_{2} a_{3}$.

Для анализа минимальных систем индекса 2 воспользуемся их классификацией из теоремы 8. Для матриц вида (5.14)

$$
\operatorname{det} M_{9}=2=2 \operatorname{det} \Gamma, \quad \Delta_{2}\left(M_{9}\right)=2-a_{3}-b_{3} \geqslant 0 .
$$

Если предположить, что матрица (5.14) задает вполне минимальную систему, то будет выполняться равенство $a_{3}=b_{3}$ (узлы $\gamma_{a}$ и $\gamma_{b}$ не должны нарушать минимальности друг друга). Вектор

$$
\frac{1}{2}\left(\gamma_{a}-\gamma_{b}-\gamma_{c}\right)=\left(0,-1, a_{3}-\frac{1}{2}\right)
$$

также не должен нарушать минимальность узлов $\gamma_{a}$ и $\gamma_{b}$. Это возможно, только если $a_{3}-$ $1 / 2<-a_{3}$, т.е. при $a_{3}<1 / 4$. При таком ограничении

$$
\Delta_{1}\left(M_{9}\right)=1-a_{3}-b_{3}=1-2 a_{3}>\frac{1}{2}>0 .
$$

Для матриц вида (5.15) также $\operatorname{det} M_{10}=2 \operatorname{det} \Gamma$. Значит,

$\Delta_{2}\left(M_{10}\right)=c_{2} b_{3}+1-a_{2} a_{3}-b_{3}+a_{2}+a_{3} c_{2}=\left(a_{2}+c_{2}\right)\left(1-a_{3}\right)+\left(1-c_{2}\right)\left(1-b_{3}\right)+2 a_{3} c_{2} \geqslant 0$.

Матрица (5.15) не может задавать вполне минимальную систему, поскольку при $a_{3}+b_{3} \geqslant 1$ вектор

$$
\frac{1}{2}\left(\gamma_{a}+\gamma_{b}-\gamma_{c}\right)=\left(1, \frac{1-a_{2}-c_{2}}{2}, \frac{-1+a_{3}-b_{3}}{2}\right)
$$

нарушает минимальность узла $\gamma_{b}=\left(1,1, b_{3}\right)$.

Для матриц вида (5.16) утверждение теоремы проверяется так же, как и для матриц вида (5.2). 
Рассмотрим теперь исключительные базисы, классифицированные в теореме 10. Оценка (6.2) следует из соотношений

$$
\Delta_{2}\left(M_{12}\right)=\left(2+c_{2}-a_{2}\right)\left(a_{3}+b_{3}-1\right)+2 b_{3}\left(2 a_{2}+c_{2}-2\right)+b_{3}\left(1-c_{2}\right)+\left(a_{2}+c_{2}\right) a_{3} \geqslant 0 .
$$

Предположим, что матрица (5.17) задает вполне минимальную систему. Вектор

$$
\gamma_{d}=\gamma_{a}+\gamma_{b}+\gamma_{c}=\left(-1,1-a_{2}-c_{2}, 1-a_{3}-b_{3}\right)
$$

не должен нарушать минимальность узла $\gamma_{b}=\left(-1,1,-b_{3}\right)$. Это возможно, если выполнено по крайней мере одно из двух эквивалентных условий: $a_{2}=1$ или $a_{3}=1$. Без ограничения общности будем считать, что $a_{2}=1$. Так как $\gamma_{a}$ и $\gamma_{b}$ не нарушают минимальности друг друга, то $a_{3}=b_{3}$ (кроме того, $a_{3}+b_{3} \geqslant 1$, т.е. $\left.a_{3}=b_{3}>0\right)$. Узел $\gamma_{c}$ не нарушает минимальность $\gamma_{a}$, следовательно, $c_{2}=1$. Теперь узел $\gamma_{d}=\left(-1,-1, c_{3}-2 a_{3}\right)$ не должен нарушать минимальность $\gamma_{c}=(-1,-1,1)$. Следовательно, $a_{3}=1$ и матрица $M\left(\gamma_{a}, \gamma_{b}, \gamma_{c}\right)$ принимает вид

$$
\left(\begin{array}{rrr}
1 & -1 & -1 \\
-1 & 1 & -1 \\
-1 & -1 & 1
\end{array}\right)
$$

Для такой минимальной системы $\Delta_{1}\left(M_{12}\right)=1$.

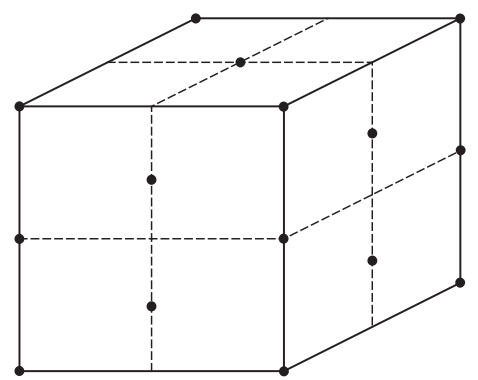

Рис. 7

ЗАмечаниЕ 3 . Константа 2 в оценке (6.2) неулучшаема. В матрицах $M_{j}(j=5,6,7,9$, $10,11,12)$ можно так подобрать коэффициенты, чтобы выполнялось равенство $\Delta_{2}\left(M_{j}\right)=0$ (для матрицы $M_{7}-$ в пределе). Во всех случаях соответствующая экстремальная решетка, с точностью до эквивалентных преобразований имеет базисную матрицу

$$
\left(\begin{array}{rrr}
0 & 1 & 1 \\
1 & 1 & -1 \\
\frac{1}{2} & 1 & -1
\end{array}\right) .
$$

На рис. 7 изображены точки экстремальной решетки на поверхности куба П.

\section{Список литературы}

[1] F. Klein, "Über eine geometrische Auffassung der gewöhnlichen Kettenbruchentwickelung", Gött. Nachr., 3 (1895), 357-359.

[2] F. Klein, "Sur une représentation géométrique du développement en fraction continue ordinaire", Nouv. Ann. (3), 15 (1896), 327-331. 
[3] Г. Вороной, Об одном обобщении алгоритма непрерывных дробей, Докт. дис., Варшава, 1896.

[4] Г. Вороной, Собрание сочинений, Т. 1, Киев, 1952.

[5] H. Minkowski, "Généralisation de la théorie des fractions continues", Ann. Sci. École Norm. Sup. (3), 13 (1896), 41-60.

[6] H. Minkowski, "Zur Theorie der Kettenbrüche", Gesammelte Abhandlungen, Band 1, B. G. Teubner, Leipzig, 1911, 278-292.

[7] В.А. Быковский, "Относительные минимумы решеток и вершины многогранников Клейна", Функи. анализ и его прил., 40:1 (2006), 69-71.

[8] О.Н. Герман, "Полиэдры Клейна и относительные минимумы решеток”, Матем. заметки, 79:4 (2006), 546-552.

[9] Б. Н. Делоне, Д. К. Фаддеев, “Теория иррациональностей третьей степени”, Тр. Матем. ин-та им. В. А. Стеклова, 11, Изд-во АН СССР, М.-Л., 1940, 3-340.

[10] Б. Н. Делоне, Петербургская школа теории чисел, Изд-во АН СССР, М.-Л., 1947.

[11] H. Hancock, Development of the Minkowski Geometry of Numbers, Vol. 1, 2, Dover Publ., New York, 1964.

[12] В. А. Быковский, “О погрешности теоретико-числовых квадратурных формул”, Докл. РАН, 389:2 (2003), 154-155.

[13] В. А. Быковский, “Отклонение сеток Коробова", Изв. РАН. Сер. матем., 76:3 (2012), 19-38.

[14] Н. М. Коробов, Теоретико-числовые методы в приближенном анализе, МЦНМО, М., 2004.

[15] М. О. Авдеева, В.А.Быковский, "Уточнение теоремы Валена для базисов Минковского трехмерных решеток", Матем. заметки, 79:2 (2006), 163-168.

[16] K. Th. Vahlen, "Über Näherungswerte und Kettenbrüche", J. Reine Angew. Math., 115:3 (1895), 221-233.

[17] А. Я. Хинчин, Цепные дроби, Наука, М., 1978.

[18] М. О. Авдеева, В.А. Быковский, “Аналог теоремы Валена для совместных приближений пары чисел", Матем. сб., 194:7 (2003), 3-14.

[19] В. А. Быковский, “Теорема Валена для двумерных подходящих дробей”, Матем. заметки, 66:1 (1999), 30-37.

[20] С. Гассан, "Структура областей Валена для трехмерных решеток", Чебышевский сб., 6:3(15) (2005), 51-84.

[21] А. В Устинов, "К трехмерной теореме Валена", Матем. заметки (в печати).

[22] В. А. Быковский, О. А. Горкуша, "Минимальные базисы трехмерных полных решеток", Матем. сб., 192:2 (2001), 57-66.

[23] Ph. Furtwängler, M. Zeisel, "Zur Minkowskischen Parallelepipedapproximation", Monatsh. Math. Phys., 30:1 (1920), 177-198.

[24] P. M. Pepper, "Une application de la géométrie des nombres à une généralisation d'une fraction continue", Ann. Sci. École Norm. Sup. (3), 56 (1939), 1-70.

[25] H. Minkowski, Diophantische Approximationen. Eine Einführung in die Zahlentheorie, B. G. Teubner, Leipzig, 1907.

[26] О.А. Горкуша, "Минимальные базисы трехмерных полных решеток", Матем. заметки, 69:3 (2001), 353-362.

[27] H. Minkowski, Gesammelte Abhandlungen, Band 2, B. G. Teubner, Leipzig, 1911. 